\title{
Modelo propuesto para la identificación y predicción de bancos en dificultades, según el contexto del Comité de Supervisión Bancaria de Basilea
}

\author{
Proposed model for the identification and prediction of weak banks, \\ in the context of the Basel Committee on Banking Supervision \\ Dulce María Gutiérrez Ramírez ${ }^{*}$,Luis Manuel Gaggero Sager², \\ Salvador Marín Hernández ${ }^{3}$, Celso Joaquín Muñoz Black ${ }^{4}$ \\ ${ }^{1}$ Universidad Nacional Autónoma de México, México \\ 2Universidad Autónoma del Estado de Morelos, México \\ ${ }^{3}$ Universidad de Murcia, España \\ ${ }^{4}$ Instituto Nacional de Electricidad y Energías Limpias, México
}

Recibido el 31 de agosto de 2015; aceptado el 23 de noviembre de 2017

Disponible en Internet el: 29 de julio de 2019

\section{Resumen}

Este trabajo propone, y describe, una investigación empírica cuyo objetivo es determinar un modelo para la identificación (la cual forma parte de las orientaciones del BCBS de 2002 y de 20151) y la predicción de bancos en dificultades, ya que la detección de una de ellas es una de las propiedades deseables de cualquier sistema de alarma (Labatut, et. al., 2009). El modelo se basa en la aplicación del análisis discriminante a nueve bancos mexicanos en 1999, año que fue clave para la estabilidad y certidumbre del sistema financiero mexicano con la publicación de la LPAB2 y la creación del IPAB3. Los indicadores financieros considerados han sido utilizados por Beaver, Altman y Ayala, y el contexto de estos indicadores está incluido actualmente dentro de los marcos de Basilea II y III4. Con la función discriminante obtenida del modelo propuesto y con base en el estudio del caso, el modelo se probó con nueve bancos mexicanos, que estuvieron en la crisis bancaria mexicana, lo cual dio un resultado del 100\% en la identificación de su respectiva situación financiera. Además, se predijo esta situación para cuatro bancos mexicanos en el

\footnotetext{
*Autor para correspondencia

Correo electrónico dgur19@ hotmail.com (D.M Gutiérrez Ramírez)

La revisión por pares es responsabilidad de la Universidad Nacional Autónoma de México.

http://dx.doi.org/10.22201/fca.24488410e.2018.1770

0186- 1042/C 2019 Universidad Nacional Autónoma de México, Facultad de Contaduría y Administración. Este es un artículo

Open Access bajo la licencia CC BY-NC-SA (https://creativecommons.org/licenses/by-nc-sa/4.0/)
} 
periodo 2000-2008 y un banco de los Estados Unidos, afectado por la crisis sub-prime, para el periodo 2000 2010; la predicción basada en los resultados empíricos concuerda, en general, con los informes anuales del IPAB y con las opiniones de los presidentes del banco estadounidense. Se concluye que el modelo propuesto puede utilizarse, adicionalmente a los modelos de alerta temprana institucionales, para predecir si un banco está o no en dificultades considerando, además de los recursos propios, la provisión para pérdidas crediticias. Es necesario señalar que, dada la complejidad matemática del análisis discriminante y a las hipótesis involucradas, se implementaron técnicas estadísticas y algoritmos para su desarrollo.

\section{Código JEL: G01, G21, C52}

Palabras clave: Bancos en dificultades, Análisis discriminante; Indicadores financieros

\section{Resumen}

This paper proposes and describes an empirical research whose objective is to determine a model for the identification (which forms part of the BCBS guidelines of 2002 and 2015 ) and the prediction of weak banks, since detection of the identification or the prediction is a property desirable of any alarm system (Labatut, et al., 2009). The model is based on the application of discriminant analysis to nine Mexican banks in 1999, a year that was key to the stability and certainty of the Mexican financial system with the publication of the LPAB and the creation of IPAB . The financial indicators considered have been used by Beaver, Altman and Ayala, and furthermore the context of this indicators is currently included into the framework of Basel II and III . With the discriminant function obtained from proposed model and with base on study of the case, the model was proved with nine Mexican banks, who were involved in the Mexican banking crisis, resulting a $100 \%$ in the identification of their respective financial situation. In addition, this situation was predicted to four Mexican banks in the period 2000-2008 and a bank from the United States, affected by the sub-prime crisis, for the period 2000 2010; the prediction based on the empirical results agrees, in general, with the annual reports of the IPAB and with the opinions of presidents of the American bank. It is concluded that the proposed model can be used, in addition to the institutional early warning models, to predict if a bank is weak or not, considering, additionally to the effect on own resources the allowance for loan losses. It should be emphasized that, given the mathematical complexity of the discriminant analysis and the hypotheses involved, we implemented statistical techniques and algorithms for their development.

JEL code: $\mathrm{G} 01, \mathrm{G} 21, \mathrm{C} 52$

Keywords: Weak banks; Discriminant analysis; Financial ratios

\footnotetext{
${ }^{1}$ Comité de Supervisión Bancaria de Basilea (Basel Committee on Banking Supervision)

${ }^{2}$ Ley para la Protección al Ahorro Bancario (Bank Savings Protection Law)

${ }^{3}$ Instituto para la Protección al Ahorro Bancario (Institute for the Protection of Banking Savings)

Conjunto integral de reformas elaborado por el BCBS para fortalecer la regulación, supervisión y gestión de riesgos del sector bancario.
} 


\section{Introducción}

En las últimas dos décadas, las crisis financieras y, en particular las bancarias, han sido más frecuentes, tanto en países en vías de desarrollo como en industrializados, y han significado una severa contracción de la producción y cuantiosas pérdidas fiscales y financieras (Morón, 2003 y Redondo y Rodríguez, 2014). Un ejemplo de ello en los países en vías de desarrollo, es la que enfrentó el sector bancario mexicano a finales de 1994, cuatro años después de su reprivatización en 1991-1992, debido a los altos índices de morosidad ocasionados por las elevadas tasas de interés, la contracción de la oferta de fondos prestables, la disminución en el nivel de intermediación financiera y la desaceleración económica, lo cual ocasionó la intervención del Estado en la capitalización de dicho sector. Por su parte, los países industrializados también han padecido dichas situaciones como lo ejemplifica la peor crisis financiera internacional de los últimos tiempos, la cual inició en agosto de 2007 y estuvo vinculada con problemas del sector inmobiliario de Estados Unidos y, particularmente, con las hipotecas de tipo sub-prime. En una economía globalizada como la actual, ningún país está exento del contagio ante una crisis tan profunda como la crisis financiera estadounidense, ya que ésta no sólo afecta al crédito hipotecario sino a todo el sistema financiero en su conjunto (Saavedra, 2008).

Para evitar las crisis bancarias sistémicas, el Foro sobre la Estabilidad Financiera (FSF, por sus siglas en inglés), sustituido por el Consejo de Estabilidad Financiera (FSB, por sus siglas en inglés) en el 2009, delegó al BCBS en el 2001 el desarrollo de orientaciones internacionales de supervisión aplicables a los bancos en dificultades, las cuales actualizó en el 2015 en respuesta a la crisis financiera de 2007 - 2009. Estas orientaciones establecen que, para identificar bancos en dificultades, los supervisores pueden emplear métodos, basados principalmente en información financiera cuantitativa, que contengan un análisis de los estados financieros y sistemas de alerta temprana (BCBS, 2001 y Redondo y Rodríguez, 2014). Con el término "banco en dificultades" nos referimos al informe, emitido por el Grupo de Trabajo en 2002, donde se establece que "un banco en dificultades es aquél cuya liquidez o solvencia está, o se verá afectada, a no ser que se produzca una gran mejoría en sus recursos financieros, perfil de riesgo, dirección estratégica de la empresa, capacidad de gestión de riesgo o gestión de calidad".

Por su parte, el Instituto para la Protección para el Ahorro Bancario (IPAB), con base en el análisis de la información financiera de las instituciones bancarias mexicanas, realizó actividades a nivel nacional permitiendo detectar anticipadamente si un banco está en dificultades. Tal es el caso del "Sistema de Alerta Temprana", que sirve para alertar sobre una situación de deterioro que pueda conducir a una potencial insolvencia o quiebra bancaria (IPAB, Informe anual 2002). 
En la década pasada, la inestabilidad de los mercados, iniciada a mediados de 2007, volvió a subrayar la importancia de la liquidez en el funcionamiento de los mercados financieros y del sector bancario. El cambio en las condiciones del mercado reveló la rapidez con que la liquidez puede agotarse y puso de manifiesto que la falta de ésta puede prolongarse durante bastante tiempo por lo que, sentando las bases de su marco de liquidez, se publicó el bcbs 144 (véase la Tabla 5). Como respuesta a esta crisis financiera internacional, surgió Basilea III (bcbs188, véase la Tabla 5), ya que Basilea I y II se centraban principalmente en el nivel de reservas que los bancos deben mantener para pérdidas bancarias.

Una de las razones por las que la crisis económica y financiera que estalló en 2007 fue tan severa, es que los sectores bancarios de numerosos países habían acumulado un apalancamiento excesivo dentro y fuera de balance (bcbs189, véase la Tabla 5). A esto se unió la gradual erosión del nivel y de la calidad de su base de capital. Al mismo tiempo, numerosos bancos mantenían niveles de liquidez insuficientes. Por todo ello, el sistema bancario no fue capaz de absorber las pérdidas sistémicas sufridas en las carteras de negociación y de crédito. La crisis se vio agravada por un proceso de desapalancamiento procíclico y por las interconexiones entre instituciones sistémicas a través de complejas operaciones. En el punto álgido de la crisis, el mercado dejó de confiar en la solvencia y liquidez de numerosas instituciones bancarias. Las deficiencias en el sector bancario rápidamente se transmitieron al resto del sistema financiero y la economía real, provocando una contracción generalizada de la liquidez y del crédito disponible. En última instancia, el sector público tuvo que intervenir con inyecciones de liquidez sin precedentes y con la provisión de capital y avales (bcbs189).

Debido a lo anteriormente expuesto, actualmente el BCBS ha establecido fuertes regulaciones a nivel internacional para evitar la severidad de las crisis económicas y financieras. Dentro de estas regulaciones consideran como instrumentos de medición a los indicadores de Alerta Temprana entre otros (bcbs144, véase la Tabla 5). Asimismo, en nuestro país se ha creado el Consejo de Estabilidad del Sistema Financiero (CESF, Informe anual, 2011) con la misma finalidad.

Por tanto, y dado que los bancos en dificultades pueden conducir o empeorar las crisis financieras, resulta evidente la necesidad de contar con una oportuna identificación, mediante metodologías de predicción (Redondo y Rodríguez 2014), de dichos bancos para tomar medidas correctivas que restauren su solidez y limiten sus pérdidas potenciales. Es por esto que en este trabajo se propone un modelo que permita identificar y predecir un banco en dificultades considerando que aun cuando la mayoría de las explicaciones de dichas dificultades son de tipo macroeconómico, también existen trabajos que las relacionan con los propios indicadores de la banca, es decir, explicaciones de tipo microeconómico (Hernández y López, 2001). 


\section{Descripción cronológica de la situación financiera de la Banca Mexicana y factores que han influido en ella durante el periodo 19822009}

El sistema bancario mexicano fue nacionalizado en 1982 y sus operaciones fueron controladas hasta 1988; año en que se inició la liberación financiera que culminó con la reprivatización del sistema bancario y con la introducción de la competencia extranjera en 1994 (López y Snowden, 2000). Asimismo, debido a diversos factores, entre los cuales se encontraba el hecho de que la $\mathrm{c}^{\circ}$ artera vencida de los bancos superaba a su capital contable, en diciembre de 1994 ocurrió una devaluación de casi un 100\% lo que, junto con una elevación de las tasas de interés al inicio de 1995, generó un desbalance cambiario y crediticio en la operación de los bancos y algunos de éstos pasaron de la quiebra técnica a la quiebra plena (Correa, 1999).

Durante el periodo 1995-1998 se aplicaron acciones para enfrentar la crisis bancaria mexicana (Turrent, 2003 y Murillo, 2005). La primera de ellas fue resolver los problemas de liquidez y la segunda resolver los problemas de solvencia. Para la liquidez, el Banco de México (BANXICO) dispuso una ventanilla de crédito en dólares para evitar que los bancos incumplieran con el pago de pasivos denominados en moneda extranjera, y para la solvencia se aplicaron cuatro programas:

1) Programa de Capitalización Temporal para los bancos (PROCAPTE).

2) Intervención de los bancos que se encontraban en una situación más crítica para procurar su recuperación; la cual permitió que las instituciones entraran al Programa de Saneamiento.

3) Un segundo Programa de Capitalización y Compra de Cartera (PCCC).

4) Programas de apoyo para los deudores de la banca. Estos programas, destinados al fortalecimiento del sector bancario mexicano, se llevaron a cabo mediante el Fondo Bancario de Protección al Ahorro (FOBAPROA) (Hernández y López, 2001).

La crisis bancaria mencionada evidenció el mecanismo de protección de los recursos de los depositantes en los bancos, ya que no fue suficiente y se requirió el apoyo del Gobierno Federal (Hazera et al., 2015) para evitar el colapso del sistema financiero y el de pagos. De esta manera, el 19 de enero de 1999 se publicó la LPAB dando origen al IPAB el 21 de mayo de 1999, el cual asumió prácticamente la totalidad de los pasivos del FOBAPROA. Este marco legal hizo posible una mayor regulación de la protección al ahorro y la necesidad de actuar en coordinación con el resto de las autoridades financieras en la ejecución de las acciones requeridas para procurar la solidez de las instituciones de banca múltiple. Asimismo, el IPAB hizo posible que se generara la adquisición o fusión de un banco con otro de mayor solvencia, así como la desaparición o capitalización de algunas instituciones bancarias. Cabe señalar que con estas medidas, aunadas a la venta de activos no estratégicos, al aumento en las reservas 
y al saneamiento de la cartera vencida, la generación de utilidades permitió mejorar, durante 1999, los índices de capitalización de la banca, los cuales superaron ampliamente el 8\% mínimo requerido por las autoridades, con base en los acuerdos de Basilea (Hernández y López, 2001). Por tanto, resulta razonable considerar el año de 1999 como idóneo para representar el espacio muestral y, según Mora, 1994, parece ser idóneo seleccionar una muestra con datos correspondientes al mismo periodo.

Con la conclusión del Nuevo Programa en 2005, el IPAB canjeó instrumentos emitidos por el FOBAPROA por nuevos títulos y dio lugar a la sustitución del PCCC en el cual participaron y sobrevivieron a la crisis financiera algunos de los bancos analizados en este trabajo.

En el año de 2007, un segmento del sistema financiero internacional cayó en una crisis intensa detonada por un incremento, en los Estados Unidos, de los índices de morosidad de los créditos hipotecarios de menor calidad conocidos como "subprime" (BANXICO, Informe Anual, 2007) lo que, a su vez, trajo consigo la quiebra de Lehman Brothers en septiembre de 2008 y, por consiguiente, la intensificación de la crisis financiera internacional y propagación de sus efectos a las economías emergentes. Así, el costo del crédito interbancario aumentó abruptamente y la liquidez en los mercados financieros cayó aún más complicándose la situación como resultado de los vínculos de retroalimentación entre el sector financiero y el sector real por lo que, además de la crisis de liquidez, los mercados financieros enfrentaron un problema de solvencia que dio lugar al riesgo de un colapso del sistema financiero internacional. En este contexto, los flujos de capital a las economías emergentes se contrajeron de manera pronunciada afectando los tipos de cambio y los mercados accionarios y de deuda de muchos de estos países. Estos hechos afectaron al sistema bancario mexicano por lo que BANXICO, en coordinación con la Secretaría de Hacienda y Crédito Público (SHCP), instrumentó diversas acciones orientadas a preservar el buen funcionamiento de los mercados financieros internos (BANXICO, Informe Anual 2008), lo cual ayudó a que la banca mexicana en su conjunto continuara generando utilidades y se encontrara bien capitalizada y con reservas crediticias suficientes para afrontar el alza de los índices de morosidad en el 2008 (BANXICO, Reporte sobre el Sistema Financiero, 2009).

\section{Clasificación de los nueve Bancos Mexicanos analizados}

Para aplicar el análisis discriminante se requiere clasificar a los individuos en grupos alternativos por lo que en la Tabla 1 se presentan algunos lineamientos del IPAB que sirvieron para clasificar los bancos en dificultades y los bancos no en dificultades. 
Tabla 1

Descripción de algunos lineamientos del IPAB y sus respectivas medidas realizadas

\section{DESCRIPCIÓN Y MEDIDAS REALIZADAS}

Tipo de programa: (2) Saneamiento (IPAB, Informe Anual 1999). Bancos insolventes cuyos accionistas no tenían la capacidad de aportar los recursos necesarios para capitalizarlos. El FOBAPROA, aportó capital, tomó el control accionario de la sociedad y realizó operaciones de saneamiento para que pudieran ser adquiridas por una entidad financiera o, de otra forma, proceder a la liquidación de sus activos. Estas instituciones fueron intervenidas previamente por la Comisión Nacional Bancaria y de Valores (CNBV), lo cual se realizó de dos maneras: una con carácter de gerencia y la otra de facto o administrativa. (Murillo, 2005). Posteriormente, el IPAB dispuso de los recursos provenientes de la recuperación de bienes para la conclusión de los Programas de Saneamiento y la Liquidación de los Programas del FOBAPROA. Así, de acuerdo con el inciso I, estos bancos se definen en este trabajo como "Bancos en dificultades".

Tipo de programa: (3) PCCC (IPAB, Informe Anual 1999). Bancos insolventes en los que la CNBV buscó que los propios accionistas o nuevos socios aportaran más dinero a través del PCCC; su insolvencia se debió a la administración deficiente, a la insuficiencia de capital o a problemas graves en la recuperación de los créditos otorgados. Estos bancos afectaron, en fideicomiso, los flujos derivados de una parte de su cartera de créditos, entregándoles a cambio pagarés suscritos por el FOBAPROA con vencimiento a 10 años; obligándose a compartir con éste las pérdidas resultantes de la cartera no recuperada (acuerdo de participación de pérdidas). Asimismo, se celebró con el FOBAPROA un esquema de incentivos mediante el cual los bancos cubrieron sus deficiencias de reservas en los fideicomisos; el Comité Técnico del FOBAPROA, determinó la insuficiencia de reservas preventivas y que los bancos cubrieran gradualmente dicho faltante con recursos propios a partir de los resultados obtenidos en la cobranza de los créditos. En el Anexo 4 del Informe de Rendición de Cuentas 2000-2005, se presentan los saldos que fueron base para la formulación de los estados financieros anuales dictaminados por el IPAB partiendo de la información financiera de los fideicomisos. Así, de acuerdo con el inciso I, estos bancos se definen en este trabajo como "Bancos no en dificultades".

(IPAB, Informe Anual 2000). En junio de 1999, en cumplimiento del Artículo Quinto Transitorio de la LPAB, el IPAB expidió las Reglas Generales del nuevo programa para proceder al intercambio de los pagarés emitidos por el FOBAPROA bajo el PCCC. En octubre de este mismo año, las instituciones que solicitaron al Instituto su adhesión al nuevo programa, entregando sus planes de consolidación financiera, un plan anual de cobranza de cartera, y el cumplimiento de los niveles de capitalización fueron: Banamex, Bancomer, Banorte, Bital y BBV. Así, la CNBV emitió su opinión favorable a dichos planes entregados. En diciembre de 1999, el Congreso de la Unión, le otorgó al IPAB la facultad de contratar créditos o emitir valores con el objeto de canjear o refinanciar sus obligaciones, y hacer frente a sus obligaciones de pago, otorgar liquidez a sus títulos y, en general, mejorar los términos y condiciones de sus obligaciones financieras.

(IPAB, Informe Anual 2000). Durante el 2000, los principales bancos que operaban en México enfocaron sus esfuerzos a fortalecer su solidez financiera acelerando sus procesos de capitalización, creando mayores reservas, reduciendo la cartera vencida y cumpliendo anticipadamente con los requerimientos de capitalización, que posteriormente regirán en el 2003.

(CNBV, Boletín Estadístico, diciembre de 2004). A partir de 2004, la CNBV analiza la información financiera de los bancos para determinar la estabilidad financiera o solvencia de éstos. Este proceso lo realiza mediante una clasificación de categorías (I a V) de alertas tempranas, tomando como base el Índice de Capitalización (ICAP) que le da a conocer el BANXICO ("Ley de Instituciones de Crédito" y "Disposiciones de carácter general aplicables a las instituciones de crédito", publicadas en el Diario Oficial de la Federación el 18-julio-1990 y el 02-diciembre-2005, respectivamente).

Fuente: Elaboración propia 
En relación con la Tabla 1 se señala que, según el IPAB (Informe Anual, 2002) y el criterio del regulador (BANXICO, Reporte sobre el Sistema Financiero, 2006), la solvencia de un banco se detecta mediante el mecanismo de "alerta temprana" con el cálculo del Índice de Capitalización (ICAP). No obstante, y en última instancia, la solvencia de un banco también depende de su capacidad para generar utilidades y del índice de cobertura de su cartera vencida con provisiones. Considerando lo expuesto en la Tabla 1 y en las acciones realizadas por los bancos, mostradas en las tablas 2 y 3 , se presenta en estas últimas la clasificación de los bancos analizados como Bancos en dificultades y los Bancos no en dificultades, respectivamente. Es necesario comentar que Altman, et al., 2014, considera una clasificación de los bancos en dificultades, y no en dificultades, muy similar a la realizada en este trabajo y Marín, et al., 2011, señala como fracaso empresarial a aquellas instituciones que fueron intervenidas por el FOBAPROA.

Tabla 2

Clasificación de los bancos en dificultades (Programa 2) y sus respectivas acciones realizadas

Banca Serfín, S. A. (1)*. Programa de Saneamiento Financiero: Intervención Administrativa

Junio/1999. El IPAB resolvió capitalizar a Banca Serfín, para que retomara el camino hacia la rentabilidad, adquiriendo la titularidad de las acciones representativas del capital social (IPAB, Informe Anual 1999).

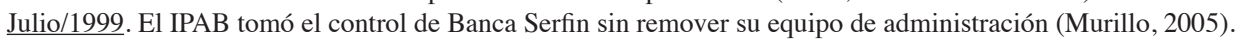
$\underline{06 / O c t u b r e / 1999}$. Se publicó la convocatoria para ceder en administración, cartera comercial, industrial e hipotecaria de Banca Serfín, con un valor aproximado de 25,000 millones de pesos (IPAB, Informe Anual 1999).

27/Octubre/1999. Banorte fue nombrado participante ganador (IPAB, Informe Anual 1999).

28/Febrero/2000. Banorte firmó el contrato de administración (IPAB, Informe Anual 1999).

02/Diciembre/1999. El IPAB, en paralelo con el proceso de saneamiento, inició el proceso de venta de las acciones de Grupo Financiero Serfín y publicó la convocatoria para la adquisición de hasta el 100 por ciento de los títulos de las acciones representativas del capital social de la institución (IPAB, Informe Anual 2000).

Mayo/2000. Grupo Financiero Santander Mexicano resultó ganador del proceso de licitación (IPAB, Informe Anual 2000).

Bancen (2). Programa de Saneamiento Financiero: Intervención Gerencial

Junio/1995. Bancen fue intervenido (Murillo, 2005).

Marzo/1996. Banorte administra Bancen (Murillo, 2005).

Junio/1997. FOBAPROA enajena a favor de Banorte las acciones de Bancen (Murillo, 2005). 
Banca Quadrum (3). Programa de Saneamiento Financiero: Intervención Gerencial y en Liquidación

Agosto/2001. Fue intervenida gerencialmente (Murillo, 2005).

11/Junio/2001. En virtud de la solicitud hecha por Quadrum, relacionada con el otorgamiento de apoyo financiero, el IPAB contrató a un tercero especializado para que realizara el estudio técnico (IPAB, Informe Anual 2001). 29/Agosto/2001. La SHCP consideró que Quadrum se ubicaba en causal de revocación de la autorización otorgada por esa Secretaría para organizarse y operar como institución de banca múltiple, ya que arrojaba pérdidas que afectaban su capital mínimo. La SHCP emplazó a esa institución para que manifestara lo que a su derecho conviniera y otorgó un plazo de sesenta días hábiles contados a partir de la fecha mencionada para que esa institución se capitalizara, toda vez que manifestaba problemas de solvencia. El plazo se prorrogó hasta el 11 de marzo de 2002 (IPAB, Informe Anual 2001).

28/Febrero/2002. Debido a que Quadrum no logró capitalizarse en el plazo otorgado por la SHCP, esta autoridad le revocó la autorización otorgada, para organizarse y operar como institución de banca múltiple, por lo que entró en liquidación (IPAB, Informe Anual 2002).

04/Marzo/2002. Se llevaron a cabo los actos de entrega-recepción de Quadrum entre el interventor gerente de la CNBV y el Instituto, y entre este último y el apoderado liquidador (IPAB, Informe Anual 2002).

2003. Se elaboraron documentos para iniciar la selección del despacho jurídico encargado de la auditoría legal a la gestión de la intervención gerencial en la Banca en Liquidación, y se dio seguimiento al desarrollo de las auditorías en materia legal a la gestión de la intervención gerencial de Banca Quadrum (IPAB, Informe Anual 2003).

Banpaís (4). Programa de Saneamiento Financiero: Intervención Gerencial

Marzo/1995. Fue intervenido gerencialmente (Murillo, 2005).

Abril/1997. Se acepta la propuesta de compra de Banorte (Murillo, 2005).

Diciembre/1997. Se celebra el contrato de compraventa. A partir de entonces se levanta la intervención y la administración la asume Banorte (Murillo, 2005).

Banco Santander Mexicano, S. A. (5). Programa de Saneamiento Financiero: Venta de Administración y Cobranza de Cartera de Crédito

Noviembre/1999. Se publicó la convocatoria para licitar la adquisición de derechos de administración y cobranza de cartera de crédito de Banco Santander Mexicano, S. A. En este caso se trató de una operación de venta y no de cesión en administración. La operación comprende la licitación de 5 paquetes de crédito comerciales e industriales, vencidos en su mayoría y con un valor aproximado de. \$7,104 millones de pesos (IPAB, Informe Anual 1999).

Marzo/2000. Se subastó una cartera de aproximadamente 19 mil 972 créditos comerciales e industriales de Banco Santander Mexicano, con un valor de principal de 6 mil 943 millones de pesos (IPAB, Informe Anual 2000).

"(Número de banco)

Fuente: Elaboración propia 
Tabla 3

Clasificación de los bancos no en dificultades (Programa 3) y sus respectivas acciones realizadas

Banorte (6)

2000. Continúa bajo el consejo de administración original (Murillo 2005).

Bancomer (7)

Agosto/2000. Bancomer se fusiona con Grupo Financiero BBV-Probursa ya que, aún cuando tenía un nivel de capitalización adecuado, requería de inversiones adicionales para poder competir en el nuevo entorno de la industria. Esta fusión fue un acuerdo entre bancos en el cual no participó el FOBAPROA (Murillo, 2005).

Banamex (8)

Agosto/2001. Se fusiona con Citibank, ya que, aún cuando tenía un nivel de capitalización adecuado, requería de inversiones adicionales para poder competir en el nuevo entorno de la industria. Esta fusión fue un acuerdo entre bancos en el cual no participó el FOBAPROA (Murillo, 2005).

HSBC; antes BITAL (9)

2000. Continúa bajo el consejo de administración original (Murillo 2005).

*(Número de banco)

Fuente: Elaboración propia

\section{Modelo de Análisis Discriminante}

Se señala que, aun cuando fue formulado por Altman en 1968, el análisis discriminante se sigue empleando en la actualidad para clasificar a entidades en grupos alternativos como puede verse en los trabajos de Crespo, 2011, y de Redondo y Rodríguez, 2014. Al parecer, el análisis discriminante ha predominado en los estudios sobre las dificultades en entidades financieras desde un punto de vista empírico y no ha perdido protagonismo, tal vez por ser una técnica bien estudiada y, sobre todo, por los buenos resultados que ha aportado (Redondo y Rodríguez, 2014). Este análisis se usa para clasificar a distintos individuos en grupos alternativos a partir de los valores de un conjunto de variables de aquéllos a los que se pretende clasificar. Así, cada individuo puede pertenecer a un solo grupo y su pertenencia se introduce en el análisis mediante una variable categórica (dependiente) que toma tantos valores como grupos existentes se tengan. Por su parte, las variables utilizadas para la clasificación de los individuos se denominan variables clasificadoras (independientes) y su información se sintetiza en funciones denominadas funciones discriminantes. El análisis discriminante puede aplicarse para fines explicativos y predictivos; en la primera aplicación (véase el inciso V) se trata de determinar la contribución de cada variable clasificadora a la clasificación correcta de cada individuo, y en la segunda (véase el inciso VI) se trata de determinar el grupo al que pertenece un individuo para el que se conocen los valores que toman las variables clasificadoras (Uriel y Aldás, 2005 y Carvallo, 2007). Las hipótesis estadísticas de este análisis son la homoscedasticidad y la normalidad multivariante de los grupos (Hair et al., 2005), las cuales se demuestran en los incisos V.1 y V.2, respectivamente. 


\section{Aplicación explicativa del Análisis Discriminante a los nueve Bancos Mexicanos}

El análisis discriminante se aplicó a los nueve Bancos Mexicanos presentados en las tablas 2 y 3 para el periodo de 1999. Así, la variable categórica (dependiente) se define como "Bancos en dificultades" (Grupo 1) ó "Bancos no en dificultades" (Grupo 2).

En lo referente a las variables clasificadoras (independientes) empleadas en este trabajo, se señala que son trece de algunos de los indicadores financieros que han sido utilizados en el análisis univariante de Beaver, 1966, en el análisis discriminante múltiple de Altman, 1968, en los modelos de alerta temprana para crisis financieras de Ayala, 1999, y cuyo contexto está actualmente contemplado dentro de los acuerdos de Basilea II y III del BCBS. La selección de dichos indicadores se realizó de acuerdo con lo propuesto por diferentes autores que han trabajado sobre la predicción de crisis empresarial o bancaria; sus comentarios específicos son:

Fondo de Maniobra.- En 1968, Altman utilizó este indicador financiero mencionando que frecuentemente éste se encuentra en los estudios de empresas con problemas financieros y, de los tres indicadores de liquidez que evaluó, lo consideró el más valioso. Asimismo, Beaver, en 1966, lo consideró como el segundo mejor para la predicción y Alfaro, Gámez y García, 2008, lo emplearon para predecir el fracaso empresarial de 1180 empresas (comerciales e industriales). Asimismo, Lifschutz, 2010, Alkhatib y Eqab, 2011, y Naresh y Sree, 2014, han publicado trabajos que indican la buena predicción de la bancarrota a través del empleo del modelo de Altman y, por tanto, el empleo de este indicador.

Rendimiento sobre Activos.- En 1968, Beaver examinó una gran cantidad de empresas fracasadas para las cuales nunca se había hecho un pronóstico de falla. Mediante este examen dedujo que este indicador tenía un buen poder de predicción. Un estudio más reciente (Cools y van Toor, 2015) ha utilizado este indicador, entre otros, para realizar un comparativo entre bancos débiles y bancos fuertes. Asimismo, Gamlath y Rathiranee, 2014, emplearon este indicador para realizar un análisis de regresión a una muestra de siete bancos comerciales en Sri Lanka en el periodo 2007-2011 para determinar la relación entre el manejo del capital de trabajo y la rentabilidad. ElAnsary y Hafez, 2015, en su estudio empírico sobre los bancos Egipcios relacionaron el Coeficiente de Suficiencia del Capital como variable dependiente con el Rendimiento sobre activos para analizar la Rentabilidad entre otras variables independientes, concluyendo que están significativamente correlacionados durante el período 2003 a 2013. Labatut, et. al., 2009, considera este indicador muy eficiente para la detección de insolvencia empresarial. Cabe señalar que el trabajo de Labatut fue realizado para pequeñas y micro empresas, pero él mismo menciona que su estudio puede ser extrapolado a otros contextos donde se reproduzcan situaciones similares; lo cual es el caso de la banca. Por su parte, Chávez y Córdova, 2017, consideraron este indicador en el estudio de la situación financiera de compañías ecuatorianas con el análisis discriminante. 
Productividad.- En 1968, Altman empleó este indicador para medir la verdadera productividad de los activos de la empresa y, dado que la existencia definitiva de éste se basa en el poder de las ganancias de sus activos, parece ser particularmente apropiado para estudios relacionados con fallas corporativas y supera a otras medidas de rentabilidad. En 1999, Carrasco señaló que, según Altman, 1968, la Productividad es fundamental, ya que la existencia de una entidad viene determinada por el poder de rentabilidad de sus activos. Por su parte, Alfaro, Gámez y García, 2008, usaron este indicador que resultó muy importante en su modelo discriminante y mencionaron que tienen más opciones de permanecer activas las empresas que cuentan con una mayor rentabilidad económica, por lo que la Productividad es menor para bancos fracasados (Laffarga, Martín y Vázquez, 1987). Asimismo, Lifschutz, 2010, Alkhatib y Eqab, 2011, y Naresh y Sree, 2014, han publicado trabajos que indican buena predicción de la bancarrota a través del empleo del modelo de Altman. Labatut, et. al., 2009 empleó también este indicador.

Apalancamiento Interno.- En 1999, Carrasco, señaló que según Altman, 1968, el indicador financiero valor de mercado de las acciones/valor contable de las deudas totales es un predictor eficaz de la bancarrota. Sin embargo, debido a que no es fácil obtener este tipo de información se decidió emplear el indicador financiero de Apalancamiento Interno como predictor. También ha sido utilizado por Millán de la Lastra et al, 2013, sobretodo en la aparición de pérdidas, particularmente en la cartera de crédito, que impacta significativamente en el deterioro de sus activos. Por tanto, debe evitarse la acumulación de apalancamiento de los bancos. Y Gutiérrez y Abad, 2014. Muestran en los resultados que la estructura patrimonial de las entidades, definida como autonomía financiera (fondos propios/pasivo), es una de las dos variables con mayor capacidad predictiva. Labatut, et. al., 2009 empleó también este indicador. Chávez y Córdova, 2017 aplicaron el inverso de este indicador.

Liquidez.- Este indicador pertenece a la categoría de Riesgo de Liquidez dentro del acrónimo MACRO (a la categoría Liquidity de CAMEL). Debido a que los bancos son intermediarios financieros cuyos pasivos son depósitos a corto plazo y sus activos son préstamos a largo plazo, el desbalance entre activos y pasivos es una consecuencia natural de la actividad financiera que, de no ser administrada adecuadamente, puede degenerar en problemas de solvencia (Ayala, 1999). Por su parte, Laffarga, Martín y Vázquez, 1991, la utilizaron como uno de los quince indicadores que seleccionaron para su análisis de predicción de quiebra bancaria en Bancos Españoles y en 2008, Alfaro, Gámez y García, también emplearon este indicador financiero. En 2010, Gamlath y Rathiranee usaron este indicador en su análisis de regresión a siete bancos comerciales en Sri Lanka en 2007-2011. Labatut, et. al., 2009 y Chávez y Córdova, 2017 aplicaron también este indicador.

Morosidad.- Este indicador pertenece a la categoría de calidad de Activos dentro de MACRO (Asset quality de CAMEL). Hernández y López, 2001, sugieren que este indicador es estadísticamente significativo para explicar las crisis bancarias y refleja el riesgo crédito. Entre 
más alta sea esta relación, la posibilidad de supervivencia del banco disminuye. Los bancos con esta situación tienen forzosamente que aportar, por las pérdidas, una porción considerable de los activos, lo que conduce a una reducción de las ganancias y, por consiguiente, del capital.

El incremento de la morosidad en el período 2007-2012 tuvo consecuencias muy graves en el sistema financiero español llevando a la desaparición de las Cajas de Ahorro, las cuales representaban el 50\% del sistema crediticio español (Climent, 2017).

Cobertura de Provisiones.- Este indicador pertenece a la categoría de calidad de Activos dentro de MACRO. Carrasco, 1999, señala que Whalen y Thomson, 1988, la usaron en modelos para predecir el deterioro en la situación del banco a través de cambios en la valoración CAMEL. Asimismo, en este sentido, Climent 2017, hizo un estudio para la predicción del comportamiento de las dotaciones para los deterioros de las entidades de crédito de España en diferentes etapas de los ciclos económicos por los que han pasado y así obtener una información más real sobre las necesidades de estas dotaciones para las provisiones por deterioro de los créditos.

Riesgo Crediticio.- Este indicador pertenece a la categoría de suficiencia de Capital dentro de MACRO (Capital de CAMEL). Carrasco, 1999, menciona que los modelos construidos por Whalen y Thomson, 1988, utilizando solamente este indicador, dan resultados tan buenos como los obtenidos por otros modelos más complicados y que esta variable parece ser sustitutiva de la calificación de los activos en la valoración CAMEL. Por su parte, Ayala, 1999, indica que las variaciones en el tipo de cambio pueden afectar significativamente la situación financiera de un banco; el riesgo de este tipo de cambio puede convertirse en riesgo crediticio si la rentabilidad de los prestatarios no tiene incorporada correctamente la devaluación esperada.

Rentabilidad sobre Activos Productivos.- Este indicador financiero pertenece a la categoría de Resultados Operativos dentro de MACRO (Earnings de CAMEL) y se utiliza para medir el riesgo inherente al proceso de transformación de plazos y puede degenerar en crisis cuando el retorno de los activos cae sistemáticamente por debajo de aquel que se paga por los pasivos (Ayala, 1999; Ballester et al., 2009).

Tasa Pasiva Implícita.- Este indicador financiero pertenece a la categoría de Riesgo de Liquidez dentro de MACRO (Liquidity de CAMEL). Al igual que el indicador financiero Liquidez, la Tasa Pasiva Implícita mide el riesgo de liquidez permitiendo evaluar el riesgo de volatilidad de los pasivos que fondean los activos (Ayala, 1999). Anastasi, Burdisso, Grubisic y Lencioni, 1998, mencionan que una tasa mayor refleja una mayor dificultad para encontrar depositantes en el mercado. Por tanto, este indicador está asociado a un mayor riesgo implícito de la entidad.

Eficiencia.- Este indicador pertenece a la categoría de Manejo (Gestión) dentro de MACRO (Management de CAMEL). Arango y Botero, 2001, señalan que para Aristóbulo de Juan, una eficiente administración puede ayudar a solventar perturbaciones macroeconómicas y, por el contrario, una deficiente conduce a los bancos a la insolvencia. Los resultados de Ríos y 
Gómez en 2015 indican que los bancos con mayores márgenes de ganancia y con los menores niveles de eficiencia técnica, son lo que más aportan a la estabilidad del sector bancario.

Productividad y Manejo de Recursos.- Estos indicadores pertenecen a la categoría de Manejo (Gestión) de MACRO y se usaron en el cálculo del índice de calificación MACRO, Ayala, 1999.

En la tabla 4 se muestran las trece variables clasificadoras, $\mathrm{X}_{1}-\mathrm{X}_{13}$, consideradas en este trabajo.

Tabla 4

Variables clasificadoras y su cálculo respectivo

(Variable) Indicador Financiero

Cálculo

Liquidez (Altman)

$\left(X_{1}\right) \quad$ Fondo de Maniobra

Capital de Trabajo / Activos Totales

$\underline{\text { Rentabilidad }}$

$\left(X_{2}\right) \quad$ Rendimiento sobre Activos

$\left(X_{3}\right) \quad$ Productividad (Altman)

Utilidades Netas / Activos Totales

Utilidades antes de Impuestos / Activos Totales

Apalancamiento

$\left(X_{4}\right) \quad$ Solvencia

Capital Contable / Pasivos Totales

Riesgo de Liquidez

$\left(X_{5}\right) \quad$ Liquidez (MACRO)

Activos Circulantes / Pasivos Circulantes

Calidad de Activos

$\left(X_{6}\right) \quad$ Morosidad

$\left(X_{7}\right) \quad$ Cobertura de Provisiones

Cartera Vencida / Cartera Total

Provisiones / Cartera Vencida

Suficiencia de Capital

$\left(X_{8}\right) \quad$ Riesgo Crediticio

Cartera Vencida / Capital

Resultados Operativos

$\left(X_{9}\right) \quad$ Rentabilidad sobre Activos Productivos

Margen Bruto Financiero / Activos Productivos Promedio

Riesgo de Liquidez

$\left(X_{10}\right) \quad$ Tasa Pasiva Implícita

Intereses Pagados / Pasivos Circulantes Promedio

Manejo

$\left(X_{11}\right) \quad$ Eficiencia

$\left(X_{12}\right) \quad$ Productividad

$\left(X_{13}\right) \quad$ Manejo de Recursos

Gastos Operativos / Margen Bruto Financiero

Gastos Operativos / Activos Productivos Promedio

Activos Productivos / Pasivos Circulantes

Fuente: Elaboración propia

Adicionalmente, la tabla 5 demuestra cómo el contexto de estos indicadores está actualmente contemplado dentro de los acuerdos de Basilea II y III, a raíz de la inestabilidad del 2007. 
Tabla 5

Regulaciones del BCBS según los acuerdos de Basilea II y III

(bcbs128)*.

Convergencia internacional de medidas y normas de capital. Junio 2006

(bcbs144)* Principios para la adecuada gestión y supervisión del riesgo de liquidez (Sound Principles). Septiembre 2008

\section{(bcbs188)*}

Basilea III: Marco internacional para la medición, normalización y seguimiento del riesgo de liquidez. Diciembre 2010

\section{(bcbs189)*}

Marco regulador global para reforzar los bancos y sistemas bancarios. Diciembre de 2010 (rev. junio de 2011)
Este documento, establece los estándares mínimos a alcanzar, que el BCBS presenta para determinar si el banco posee capital suficiente para cubrir los distintos riesgos y si satisface los objetivos de suficiencia de capital establecidos. El BCBS estima que el componente esencial (básico) del capital en el que deberá hacerse mayor énfasis será el capital social en acciones y las reservas declaradas. Al capital regulador se incluirá o se deducirá la diferencia entre la provisiones (ya sean específicas, genéricas o generales para una determinada cartera) y las pérdidas esperadas

La gestión del riesgo de liquidez reviste extraordinaria importancia porque la falta de liqui$d e z$ de una sola institución puede repercutir en todo el sistema. Los indicadores de alerta temprana deberán identificar cualquier tendencia negativa o aumentos del riesgo asociados a una determinada línea de producto, como una creciente morosidad. Aun cuando el capital no es la solución a un insuficiente volumen de liquidez, ni una solución de largo plazo a la ineficacia de los procesos de gestión del riesgo, los bancos deben operar con mayores niveles de capital ya que la posición de ello puede influir en su capacidad para obtener liquidez, en especial durante una crisis.

El BCBS ha reforzado su marco de liquidez introduciendo dos estándares mínimos para la liquidez de financiación: el Coeficiente de cobertura de liquidez (Liquidity Coverage Ratio LCR) y el Coeficiente de financiación estable neta (Net Stable Funding Ratio NSFR). El primero de ellos está definido como Fondo de Activos Líquidos de Alta Calidad / Salidas de Efectivo Netas Totales durante los siguientes 30 días naturales. Entró en vigor el 01-enero-2015. Por su parte, el segundo está definido como Cantidad de Financiación Estable Disponible (ASF) / Cantidad de Financiación Estable Requerida (RSF), el cual se convertirá en estándar mínimo el 01-enero-2018.

Para elevar la resistencia del sector bancario, el BCBS ha reforzado el marco de capital regulador a partir de los tres pilares del marco de Basilea II, aumentando tanto la calidad como la cantidad de la base de capital regulador y mejoran la cobertura de riesgo del marco de capital. A ello se suma un coeficiente de apalancamiento que actúa como respaldo de las medidas de capital basadas en el riesgo, diseñado para prevenir el exceso de apalancamiento en el sistema bancario y proporcionar mayor protección frente al riesgo de modelos y errores de medición.

Uno de los principales factores desestabilizadores durante la crisis fue la incapacidad de captar correctamente los mayores riesgos dentro y fuera de balance, así como las exposiciones relacionadas con derivados.

Cabe mencionar que las iniciativas en materia de dotación de provisiones se centran en reforzar el sistema bancario frente a las pérdidas esperadas, mientras que las medidas de capital se orientan hacia las pérdidas inesperadas.
$\left.X_{4}\right),\left(X_{6}\right)$ $\left(X_{7}\right),\left(X_{8}\right)$, $\left(X_{9}\right),\left(X_{10}\right)$, $\left(X_{11}\right)$ y $\left(X_{12}\right)$

$\left(X_{1}\right),\left(X_{4}\right)$, $\left(X_{5}\right),\left(X_{6}\right)$, $\left(X_{9}\right),\left(X_{10}\right)$, $\left(X_{12}\right)$ y $\left(X_{13}\right)$

$\left(X_{1}\right),\left(X_{4}\right)$, $\left(X_{5}\right),\left(X_{9}\right)$, $\left(X_{10}\right),\left(X_{12}\right)$ y $\left(X_{13}\right)$

$\left(X_{4}\right),\left(X_{6}\right)$, $\left(X_{7}\right),\left(X_{8}\right)$, $\left(X_{9}\right),\left(X_{12}\right)$ y $\left(X_{13}\right)$ 


\section{M. Gutiérrez Ramírez, et al. / Contaduría y Administración 64(4), 2019, 1-37}

http://dx.doi.org/10.22201/fca.24488410e.2018.1770

(bcbs238)* Coeficiente de cobertura de liquidez (LCR) y herra- fondo adecuado de activos líquidos de alta calidad (High Quality Liquid Assets HQLA) mientas de seguimiento y libres de cargas, que pueden convertirse fácil e inmediatamente en efectivo en los del riesgo de liquidez mercados privados.

Enero 2013

(bcbs258)*. El marco regulador: equilibrio entre sensibilidad al riesgo, sencillez y comparabilidad Julio de 2013

$(\mathrm{d} 295)^{*}$. Coeficiente de Financiación Estable Neta (NSFR). Oct. 2014

(d311)*. Orientaciones sobre la contabilidad de pérdidas crediticias esperadas. Feb 2015

$\underline{(\mathrm{d} 350)^{*}}$.

Orientaciones sobre riesgo de crédito y contabilidad de pérdidas crediticias esperadas Dic. 2015

$(\mathrm{d} 365)^{*}$. Revisión del marco del coeficiente de apalancamiento. Abril de 2016 (revisado 25 abril 2016)

(d368)* Riesgo de tasas de interés en la cartera de inversión Abril de 2016
El BCBS sostiene que un régimen de capital basado en el riesgo debe continuar siendo la base del marco regulador para los bancos, que a su vez debe estar respaldado por medidas de liquidez y financiación y por otras medidas como el coeficiente de apalancamiento. Un coeficiente de apalancamiento da la medida en la que una cartera de activos está respaldada con capital pero no es sensible al riesgo.

El NSFR es una de las reformas esenciales del BCBS para promover un sector bancario más resiliente. El NSFR exigirá a los bancos mantener un perfil de financiación estable en relación con la composición de sus activos y actividades fuera de balance.

Establece los requisitos supervisores para prácticas de riesgo de crédito asociadas a la continua implementación y aplicación de los modelos contables para pérdidas crediticias esperadas (ECL). Uno de los principios que rigen estos requisitos es que los bancos deben contar con políticas y procesos adecuados para una identificación y gestión tempranas de los activos dudosos y para el mantenimiento de suficientes provisiones y reservas.

El Comité está de acuerdo con la Norma Internacional de Información Financiera (NIIF) 9 , Instrumentos Financieros, en que la morosidad es un indicador retardado de un incremento significativo del riesgo de crédito Los bancos deben contar con procesos de evaluación y gestión del riesgo de crédito que garanticen que este riesgo se detecta mucho antes de que las exposiciones entren en mora.

Una causa fundamental de la crisis financiera mundial fue la acumulación de excesivo apalancamiento en el sistema bancario, tanto dentro como fuera del balance. En muchos casos, los bancos se apalancaron en exceso mientras aparentemente mantenían sólidos coeficientes de capital en función del riesgo. En el punto álgido de la crisis, los mercados financieros forzaron al sector bancario a reducir su apalancamiento de tal forma que acentuaron las presiones bajistas sobre los precios de los activos.

El riesgo de tasa de interés en la cartera de inversión (Interest rate risk in the banking book IRRBB) se refiere al riesgo actual o futuro para el capital o las ganancias del banco a raíz de fluctuaciones adversas de las tasas de interés que afecten a las posiciones de su cartera de inversión. Las variaciones de las tasas de interés también afectan a las ganancias del banco al alterar los ingresos y gastos sensibles a ellas, afectando a sus ingresos netos por intereses (NII). Un IRRBB excesivo puede amenazar considerablemente la base de capital actual del banco y/o sus ganancias futuras si no se gestiona adecuadamente.
$\left(X_{1}\right),\left(X_{5}\right)$ $\mathrm{y}\left(X_{10}\right)$

$\left(X_{4}\right),\left(X_{6}\right)$, $\left(X_{7}\right),\left(X_{8}\right)$, $\left(X_{9}\right),\left(X_{12}\right)$ y $\left(X_{13}\right)$

$\left.X_{1}\right),\left(X_{4}\right)$, $\left(X_{5}\right),\left(X_{9}\right)$, $\left(X_{12}\right)$ y $\left(X_{13}\right)$

$\left(X_{6}\right),\left(X_{7}\right)$ y $\left(X_{8}\right)$

$\left(X_{6}\right),\left(X_{7}\right)$ $\mathrm{y}\left(X_{8}\right)$

$\left(X_{6}\right)$

$\left(X_{7}\right)$

$\left(X_{8}\right)$

$\left(X_{9}\right)$

$\left(X_{12}\right)$

$\left(X_{13}\right)$

$\left(X_{2}\right)$

$\left(X_{3}\right)$

$\left(X_{4}\right)$

$\left(X_{9}\right)$

$\left(X_{10}\right)$

$\left(X_{11}\right)$

"(Número de documento)

Fuente: Elaboración propia 
La tabla 6 muestra el análisis contable; parte de éste se hizo según Marín y Martínez, 2002.

Tabla 6

Valores de las variables independientes consideradas para el análisis discriminante (Año de 1999)

\begin{tabular}{|c|c|c|c|c|c|c|c|c|c|}
\hline \multirow{3}{*}{ 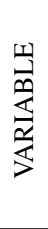 } & \multicolumn{9}{|c|}{ CLASIFICACIÓN DE LOS BANCOS } \\
\hline & \multicolumn{5}{|c|}{ BANCOS EN DIFICULTADES (Grupo 1) } & \multicolumn{4}{|c|}{$\begin{array}{l}\text { BANCOS NO EN DIFICULTADES } \\
\text { (Grupo 2) }\end{array}$} \\
\hline & $X_{1,1}^{1}{ }^{(*)}$ & $\begin{array}{c}2 \\
X_{2,1}\end{array}$ & $\begin{array}{c}3 \\
X_{3,1}\end{array}$ & $\begin{array}{c}4 \\
X_{4,1}\end{array}$ & $\begin{array}{c}5 \\
X_{5,1}\end{array}$ & $\begin{array}{c}6 \\
X_{1,2}\end{array}$ & $\begin{array}{c}{ }^{7} \\
X_{2,2}\end{array}$ & $\begin{array}{c}8 \\
X_{3,2}\end{array}$ & $\begin{array}{c}9 \\
X_{4,2}\end{array}$ \\
\hline$X_{1}$ & -0.210 & -0.131 & -0.121 & -0.222 & -0.061 & 0.049 & -0.191 & -0.120 & -0.092 \\
\hline$X_{2}$ & -0.023 & 0.010 & -0.035 & 0.006 & 0.010 & 0.012 & 0.010 & 0.024 & 0.004 \\
\hline$X_{3}$ & -0.068 & 0.011 & -0.046 & 0.007 & 0.017 & 0.010 & 0.006 & 0.025 & 0.006 \\
\hline$X_{4}$ & 0.054 & 0.077 & 0.112 & 0.046 & 0.091 & 0.091 & 0.105 & 0.142 & 0.059 \\
\hline$X_{5}$ & 0.531 & 0.503 & 0.581 & 0.429 & 0.796 & 1.121 & 0.520 & 0.712 & 0.840 \\
\hline$X_{6}$ & 0.064 & 0.048 & 0.175 & 0.040 & 0.013 & 0.087 & 0.103 & 0.117 & 0.115 \\
\hline$X_{7}$. & -1.564 & -1.447 & -0.521 & -1.157 & -1.859 & -0.751 & -0.800 & -0.992 & -0.849 \\
\hline$X_{8}$ & 0.982 & 0.584 & 1.116 & 0.743 & 0.113 & 0.458 & 0.767 & 0.618 & 0.984 \\
\hline$X_{9}$ & 0.027 & 0.059 & 0.002 & 0.074 & 0.058 & 0.084 & 0.078 & 0.094 & 0.086 \\
\hline$X_{10}$ & 0.527 & 0.852 & 0.429 & 0.497 & 0.773 & 0.390 & 0.418 & 0.350 & 0.417 \\
\hline$X_{11}$ & 1.734 & 0.866 & 36.475 & 1.037 & 0.927 & 0.864 & 0.859 & 0.638 & 1.052 \\
\hline$X_{12}$ & 0.046 & 0.051 & 0.078 & 0.077 & 0.054 & 0.072 & 0.067 & 0.060 & 0.091 \\
\hline$X_{13}$ & 2.175 & 3.644 & 2.418 & 2.474 & 3.175 & 2.102 & 2.121 & 2.115 & 1.573 \\
\hline
\end{tabular}

*(Vector de Observaciones; esta columna corresponde al vector de observaciones del elemento 1 del Grupo 1). Fuente: Elaboración propia

Con los valores de la tabla 6 y mediante las ecuaciones 1(a)-1(c) se obtuvieron los vectores de Observaciones, de Medias de los Grupos y el vector de Medias Totales, respectivamente. En dichas ecuaciones, $x_{i, g, k}$ (véase la tabla 6) es la observación de la variable $k$ del elemento $i$ del grupo $g, G$ el número de grupos, $n_{g}$ el número de elementos del grupo $g, K$ el número de variables clasificadoras y $n=n_{1}+n_{2}+\times \times \times \times+n_{\mathrm{g}}$ el tamaño de la muestra. En nuestro caso, $G=2, n_{1}=4, n_{2}=5, n=n_{1}+n_{2}=9$ y $K=13$.

$$
X_{i, g}=\left\{\begin{array}{c}
x_{i, g, 1} \\
\vdots \\
x_{i, g, K}
\end{array}\right\} i=1,2, \ldots, n_{g}, g=1,2, \ldots, G \quad X \cdot_{\cdot g}=\left\{\begin{array}{c}
\sum_{i=1}^{n_{g}} x_{i, g, 1} \\
n_{g} \\
\vdots \\
\sum_{g} x_{i, g, K} \\
n_{g}
\end{array}\right\} X=\left\{\begin{array}{c}
\sum_{g=1}^{G} \sum_{i=1}^{n_{g}} x_{i, g, 1} \\
n \\
\vdots \\
\sum_{g=1}^{G} \sum_{i=1}^{n_{g}} x_{i, g, K} \\
n
\end{array}\right\}
$$

(a)

(b) (c) 
En la tabla 7 se muestran los vectores de Medias de los Grupos y el vector de Medias Totales.

Tabla 7

Vectores de Medias de los Grupos y vector de Medias Totales

\begin{tabular}{ccc}
\hline \multicolumn{2}{c}{ Medias de los Grupos } & Medias Totales \\
$X_{\bullet}$ & $\boldsymbol{X}_{\bullet}$ & $\bar{X}$ \\
\hline$-1.489 \times 10^{-1}$ & $-8.848 \times 10^{-2}$ & $-1.221 \times 10^{-1}$ \\
$-6.219 \times 10^{-3}$ & $1.253 \times 10^{-2}$ & $2.113 \times 10^{-3}$ \\
$-1.582 \times 10^{-2}$ & $1.194 \times 10^{-2}$ & $-3.486 \times 10^{-3}$ \\
$7.617 \times 10^{-2}$ & $9.938 \times 10^{-2}$ & $8.648 \times 10^{-2}$ \\
$5.683 \times 10^{-1}$ & $7.985 \times 10^{-1}$ & $6.706 \times 10^{-1}$ \\
$6.804 \times 10^{-2}$ & $1.056 \times 10^{-1}$ & $8.475 \times 10^{-2}$ \\
$-1.310 \times 10^{0}$ & $-8.480 \times 10^{-1}$ & $-1.104 \times 10^{0}$ \\
$7.076 \times 10^{-1}$ & $7.068 \times 10^{-1}$ & $7.073 \times 10^{-1}$ \\
$4.390 \times 10^{-2}$ & $8.544 \times 10^{-2}$ & $6.236 \times 10^{-2}$ \\
$6.159 \times 10^{-1}$ & $3.940 \times 10^{-1}$ & $5.172 \times 10^{-1}$ \\
$8.208 \times 10^{0}$ & $8.531 \times 10^{-1}$ & $4.939 \times 10^{0}$ \\
$6.111 \times 10^{-2}$ & $7.247 \times 10^{-2}$ & $6.616 \times 10^{-2}$ \\
$2.777 \times 10^{0}$ & $1.978 \times 10^{0}$ & $2.422 \times 10^{0}$ \\
\hline
\end{tabular}

Fuente: Elaboración propia

\section{Hipótesis de Homoscedasticidad}

La hipótesis nula es que las matrices de covarianzas de los dos grupos son iguales, en tanto que la hipótesis alternativa es que dichas matrices no son iguales. Así, para contrastar la hipótesis nula se usó la prueba de Van Valen, la cual requiere de la distribución $t$ de Student y de ciertos valores, dependientes de la estandarización de los datos, dados por (Acuña, 2000):

$$
d_{i, g}=\sqrt{\sum_{k=1}^{K}\left(x s_{i, g, k}-M_{g, k}\right)^{2}}
$$

Donde $x s_{i, g, k}$ son las observaciones estandarizadas, y $M_{g, k}$ es la Mediana de la $k$-ésima variable estandarizada en el $g$-ésimo Grupo (Acuña, 2000). Los resultados obtenidos se muestran en la tabla 8 , y en él se observa que el $p$ valor $(0.10)$ es mayor que 0.05 , lo cual indica que se acepta la hipótesis de igualdad de matrices de covarianza. 
Tabla 8

Resultados de la prueba de Van Valen

\begin{tabular}{cccccccccc}
\hline Valor & $n_{g}$ & Media & $\begin{array}{c}\text { Desviación } \\
\text { Estándar }\end{array}$ & $\begin{array}{c}\text { Error Estándar } \\
\text { de la Media }\end{array}$ & $\begin{array}{c}\text { Diferencia } \\
\text { de Medias }\end{array}$ & $\begin{array}{c}\text { Intervalo de } \\
\text { Confianza al 95\% }\end{array}$ & g. 1. & $t$-valor & $p$-valor \\
\hline$d_{i, 1}$ & 5 & 3.592 & 1.465 & 0.655 & & & & \\
$d_{i, 2}$ & 4 & 2.135 & 0.434 & 0.217 & 1.457 & $(-0.459,3.374)$ & 4 & 2.111 & 0.10 \\
\hline
\end{tabular}

Fuente: Elaboración propia

\section{Hipótesis de Normalidad Multivariante de los grupos}

La hipótesis nula es $H_{0}: x_{g} \approx N\left(\mu_{g}, \sigma_{g}\right)$, y la alternativa es $H_{1}: x_{g} N o \approx N\left(\mu_{g}, \sigma_{g}\right)$.

Debido a la complejidad del análisis de normalidad en el caso multivariante, se usó la prueba de normalidad variable a variable para contrastar la hipótesis de los dos grupos (Uriel y Aldás, 2005). Así, dado que las muestras son pequeñas, se empleó la prueba de Shapiro \& Wilk (González, 2006) que consiste en comparar el estadístico:

$$
W_{c a l}=\frac{b^{2}}{\sum_{i=1}^{n}\left(X_{i}-\bar{X}\right)^{2}}
$$

con un valor $W_{t a b}$. Si $W_{c a l}$ es mayor que $W_{t a b}$ para un cierto nivel de significancia, se acepta la hipótesis de normalidad de los datos. Con base en esto, en la tabla 9 se presentan los resultados obtenidos para las trece variables independientes y en él se observa que solo cuatro variables no cumplen con esta hipótesis. Sin embargo, Mora, 1994, menciona que diversos autores demuestran que no es preciso que se cumpla la condición de normalidad para obtener buenos resultados. Asimismo, Redondo y Rodríguez, 2014, señalan que la dificultad existente en la práctica para que se cumplan los restrictivos supuestos de partida del análisis discriminante puede no ser un inconveniente tan grave como podría parecer a primera vista, dada su relativa robustez ante el incumplimiento de los mismos. 
Tabla 9

Prueba de Shapiro \& Wilk para la normalidad de los datos

\begin{tabular}{ccc}
\hline & & \multicolumn{2}{c}{$\begin{array}{c}W_{\text {tab }} \\
\text { Variable }\end{array}$} & $\begin{array}{c}\text { Nivel de significancia } \\
\text { cal }\end{array}$ & $\begin{array}{c}\text { ¿Se acepta o no la hipótesis de } \\
\text { normalidad? }\end{array}$ \\
\hline$X_{1}$ & 0.924 & Se acepta \\
$X_{2}$ & 0.820 & No se acepta \\
$X_{3}$ & 0.743 & No se acepta \\
$X_{4}$ & 0.960 & Se acepta \\
$X_{5}$ & 0.898 & Se acepta \\
$X_{6}$ & 0.974 & Se acepta \\
$X_{7}$ & 0.950 & Se acepta \\
$X_{8}$ & 0.956 & Se acepta \\
$X_{9}$ & 0.877 & Se acepta \\
$X_{10}$ & 0.808 & No se acepta \\
$X_{11}$ & 0.411 & No se acepta \\
$X_{12}$ & 0.964 & Se acepta \\
$X_{13}$ & 0.887 & Se acepta
\end{tabular}

Fuente: Elaboración propia

Descomposición de la Matriz de Covarianzas

Definidos los vectores de Observaciones, de Medias de los Grupos y de Medias Totales, se puede obtener la matriz de variación entre-grupos mediante la matriz de la suma de cuadrados y de productos cruzados del factor (SCPCF). Así,

$$
[F]=\sum_{g=1}^{G} n_{g}\left\{\bar{X}_{\cdot g}-\bar{X} \bigcup_{X_{\bullet g}}-\bar{X}\right\}^{T}
$$

Por su parte, la matriz de variación intra-grupos, definida como la matriz de la suma de cuadrados y productos cruzados residual (SCPCR), es:

$$
[W]=\sum_{g=1}^{G} \sum_{i=1}^{n_{g}}\left\{X_{i, g}-X_{\cdot g}\right\}\left\{X_{i, g}-X_{\cdot g}\right\}
$$

La suma de estas dos últimas matrices da como resultado la matriz de la suma de cuadrados y productos cruzados total (SCPCT). Así,

$$
[T]=[F]+[W]
$$




\section{Selección de Variables Discriminantes}

La selección de las variables que más contribuyeron a la discriminación de los grupos se hizo con el método paso a paso (Ferrán, 2001) hacia adelante y el criterio de la Lambda de Wilks para medir la potencia discriminante ganada/pérdida al introducir/sacar una variable del conjunto. En cada paso, una variable clasificadora puede entrar si su correspondiente estadístico, Fparaentrar, es mayor que un valor $F$ mínimo prefijado (Fmínentrada) o puede salir si su correspondiente estadístico, Fparasalir, es menor que un valor $F$ máximo prefijado (Fmáxsalida). El estadístico $F$ es:

$$
F=\frac{n-G-q}{G-1}\left(\frac{\Lambda_{q}}{\Lambda_{q+1}}-1\right) \text { con } n-G-q \quad \text { y } \quad G-1 \text { grados de libertad }
$$

Donde $\Lambda_{q}=\frac{\left|W_{q}\right|}{\left|T_{q}\right|}$ es la lambda de Wilks basada en las q primeras variables seleccionadas.

Para nuestro caso se especificó un Fmínentrada de 2.58 y un Fmáxsalida de 2.43. La Tabla 10 muestra las variables clasificadoras seleccionadas, por orden de aparición y siguiendo el método paso a paso hacia adelante y el criterio de la Lambda de Wilks. Aun cuando no se presentan los resultados, ninguna de las variables seleccionadas fue eliminada del conjunto de discriminación.

Tabla 10

Variables clasificadoras seleccionadas

\begin{tabular}{|c|c|c|c|c|c|c|c|}
\hline Paso & Variable & Indicador Financiero & $\begin{array}{c}\text { Lamda de } \\
\text { Wilks }\end{array}$ & $F$ & $\mathrm{gl}_{1}$ & $\mathrm{gl}_{2}$ & Sig. \\
\hline 0 & $X_{9}$ & Rentabilidad sobre Activos Productivos & 0.478 & 7.655 & 1 & 7 & 0.028 \\
\hline 1 & $X_{6}$ & Morosidad & 0.139 & 14.553 & 1 & 6 & 0.009 \\
\hline 2 & $X_{11}$ & Eficiencia & 0.092 & 2.589 & 1 & 5 & 0.169 \\
\hline 3 & $X_{5}$ & Liquidez $(M A C R O)$ & 0.048 & 3.723 & 1 & 4 & 0.126 \\
\hline
\end{tabular}

Fuente: Elaboración propia

\section{Función Discriminante Canónica}

Las funciones discriminantes canónicas están definidas como (Pérez, 2005):

$$
D_{i}=u_{i, 1} X_{1}+u_{i, 2} X_{2}+\cdots+u_{i, q} X_{q}+c_{i}
$$


Donde $i=\operatorname{mín}(G-1, q)$, siendo $q$ el número de variables clasificadoras seleccionadas. En nuestro caso $i=\operatorname{mín}(1,4)=1$, lo que significa que hay una sola función discriminante. Por tanto:

$$
D_{1}=\{u\}_{1}^{T}\{X\} \quad \text { Donde }\{u\}_{1}=\left\{\begin{array}{c}
u_{1,9} \\
u_{1,6} \\
u_{1,11} \\
u_{1,5}
\end{array}\right\} y\{X\}=\left\{\begin{array}{c}
X_{9} \\
X_{6} \\
X_{11} \\
X_{5}
\end{array}\right\}
$$

Considerando las matrices $[F],[W]$ y $[T]$ del análisis de la varianza múltiple, el vector $\{u\}_{1}$ se obtiene maximizando $\lambda_{1}$, dada por:

$$
\lambda_{1}=\frac{\text { Variabilidad entre - grupos }}{\text { Variabilidad intra - grupos }}=\frac{\{u\}_{1}^{T}[F]\{u\}_{1}}{\{u\}_{1}^{T}[W]\{u\}_{1}}
$$

La maximización de $\lambda_{1}$ conduce al problema de valores característicos siguiente:

$$
\left.[W]^{-1}[F]\{u\}_{1}=\lambda_{1}\{u\}_{1} \quad \Rightarrow \quad\{W]^{-1}[F]-\lambda_{1}[I]\right\}\{u\}_{1}=\{0\}
$$

Donde, con las variables clasificadoras seleccionadas, las matrices $[F]$ y $[W]$ son:

$$
[F]=\left[\begin{array}{rrrr}
0.004 & 0.003 & -0.679 & 0.021 \\
0.003 & 0.003 & -0.615 & 0.019 \\
-0.679 & -0.615 & 120.194 & -3.763 \\
0.021 & 0.019 & -3.763 & 0.118
\end{array}\right] \quad y \quad[W]=\left[\begin{array}{rrrr}
0.004 & -0.006 & -1.497 & -0.001 \\
-0.006 & 0.016 & 3.791 & -0.011 \\
-1.497 & 3.791 & 999.354 & 0.455 \\
-0.001 & -0.011 & 0.455 & 0.268
\end{array}\right]
$$

La ecuación 11 se resolvió mediante la descomposición de Cholesky de la matriz [W] y el algoritmo del método de potencia (Burden y Faires, 1985). Así, el valor característico $\lambda_{1}$ resultó ser igual a 20.0173 y el vector característico resultó ser:

$$
\{u\}_{1}=\left\{\begin{array}{r}
28.0289 \\
42.1010 \\
-0.1223 \\
2.4827
\end{array}\right\}
$$


Normalizando el vector $\{u\}_{1}$, de manera que $\{u\}_{1}^{T}[W]\{u\}_{1}=1$, obtenemos el vector $\{v\}_{1}$. Así,

$$
\{v\}_{1}=\left\{\begin{array}{r}
19.9415 \\
29.9533 \\
-0.0870 \\
1.7663
\end{array}\right\}
$$

Por su parte, el vector de coeficientes estandarizados de la función discriminante canónica, $\left\{u_{e}\right\}_{1}$, se obtiene con:

$$
\left\{u_{e}\right\}_{1}=[S]\{v\}_{1}
$$

Donde la matriz $[S]$ es una matriz diagonal cuyos elementos son las raíces cuadradas de los elementos de la diagonal principal de la matriz $[W]$. Por tanto,

$$
\left\{u_{e}\right\}_{1}=\left\{\begin{array}{r}
1.1809 \\
3.8127 \\
-2.7507 \\
0.9138
\end{array}\right\}
$$

El vector de estructura, el cual contiene las correlaciones intra-grupo combinadas entre las variables discriminantes canónicas estandarizadas, es:

$$
[S]^{-1}[W]\{v\}_{1}=\left\{\begin{array}{r}
0.2337 \\
0.0984 \\
-0.0775 \\
0.1483
\end{array}\right\}
$$

Por su parte, el vector de coeficientes no estandarizados de la función discriminante canónica es:

$$
\left\{u_{n e}\right\}_{1}=(n-G)^{1 / 2}\{v\}_{1}=(9-2)^{1 / 2}\left\{\begin{array}{r}
19.9415 \\
29.9533 \\
-0.0870 \\
1.7663
\end{array}\right\}=\left\{\begin{array}{r}
52.7603 \\
79.2489 \\
-0.2302 \\
4.6733
\end{array}\right\}
$$


La constante $c_{1}$ de la función discriminante canónica (Ecuación 8) con coeficientes no estandarizados se obtiene mediante la expresión:

$$
c_{1}=\{\bar{X}\}^{T}\left\{u_{n e}\right\}_{1}
$$

Donde $\{\bar{X}\}^{T}$ es el vector transpuesto de medias totales de las variables clasificadoras seleccionadas. Así, $c_{1}=-12.0032$ y la función discriminante canónica con coeficientes no estandarizados es:

$$
D_{i}=52.7603 X_{9}+79.2489 X_{6}-0.2302 X_{11}+4.6733 X_{5}-12.0032
$$

Por su parte, la i-ésima correlación canónica vale

$$
C R_{i}=\sqrt{\frac{\lambda_{i}}{1+\lambda_{i}}}=\sqrt{\frac{20.0173}{1+20.0173}}=0.976
$$

Para saber si nuestra función discriminante es significativa, contrastaremos la siguiente hipótesis nula en el $(k+1)$-ésimo paso (el proceso comienza con $k=0$ ):

$$
\mathrm{H}_{0}: \lambda_{k+1}=\lambda_{0+1}=\lambda_{1}=0
$$

mediante el estadístico de contraste (Salvador, 2000):

$$
T=\left(n-1-\frac{q+G}{2}\right) \sum_{j=k+1}^{\min (G-1, q)} \ln \left(1+\lambda_{j}\right)
$$

El cual se distribuye como una $\chi^{2}$ con $(q-k)(G-k-1)$ grados de libertad si $\mathrm{H}_{0}$ es verdad.

Por su parte, el estadístico $T$ corresponde a una lambda de Wilks dada por la Ecuación 17.

$$
\Lambda=\frac{|W|}{|T|}
$$

El $p$-valor asociado al contraste está dado por:

$$
P\left\lfloor\chi_{(q-k)(G-k-1)}^{2} \geq T_{\text {obs. }}\right\rfloor
$$


Donde $T_{o b s}$ es el valor observado de $T$. Así, Sustituyendo los valores correspondientes, se tiene:

$$
\begin{gathered}
\Lambda=0.048, T_{\text {obs. }}=\left(9-1-\frac{4+2}{2}\right) \sum_{j=1}^{1} \ln \left(1+\lambda_{j}\right)=(5) \ln (1+20.0173)=15.227, \mathrm{y} \\
P\left[\chi_{(4-0)(2-0-1)}^{2} \geq T_{\text {obs. }}\right\rfloor=P\left[\chi_{4}^{2} \geq 15.227\right]=0.004
\end{gathered}
$$

Con la correlación canónica (0.976) y el $p$-valor (0.004) se concluye que la función obtenida tiene un alto poder discriminante y es significativa. Asimismo, el cuadrado de la correlación canónica (0.953) nos hace concluir que el $95.3 \%$ de la varianza de la variable dependiente puede ser explicado por este modelo que incluye cuatro variables independientes $\left(X_{9}, X_{6}, X_{11}\right.$ y $\left.X_{5}\right)$.

Función Discriminante Lineal de Fisher

Esta función se puede obtener, para cada uno de los grupos, empleando:

$b_{i, j}=(n-G) \sum_{i=1}^{q} w_{i, l}^{*} X_{l, j} \quad i=1,2, \ldots, q$ y $j=1,2, \ldots, G$ para los coeficientes y

$a_{j}=\ln \left(\pi_{j}\right)-\frac{1}{2} \sum_{i=1}^{q} b_{i, j} \bar{X}_{i, j} \quad j=1,2, \ldots, G$ para las constantes

En las ecuaciones 19 y $20, w_{i, l}^{*}$ son los elementos de la matriz $[W]^{-1}, \bar{X}_{l, j}$ son los elementos del vector $X_{\bullet j}$ que corresponden a las variables clasificadoras seleccionadas y $\boldsymbol{\pi}_{j}$ son las probabilidades a priori. Así, para el caso en estudio, tenemos que:

Grupo 1

$$
\left\{\begin{array}{l}
X_{1,1} \\
X_{2,1} \\
X_{3,1} \\
X_{4,1}
\end{array}\right\}=\left\{\begin{array}{l}
4.390 \times 10^{-2} \\
6.804 \times 10^{-2} \\
8.208 \times 10^{0} \\
5.683 \times 10^{-1}
\end{array}\right\} \text { y } \pi_{1}=0.5
$$

Grupo 2

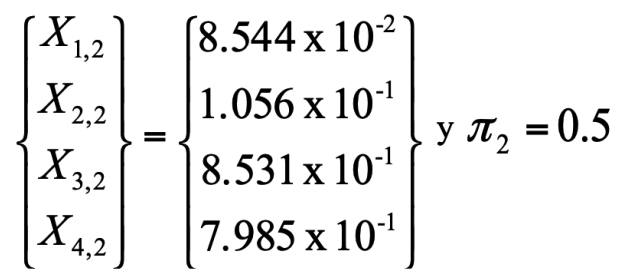




$$
\begin{aligned}
& \Rightarrow\left\{\begin{array}{l}
b_{1,1} \\
b_{2,1} \\
b_{3,1} \\
b_{4,1}
\end{array}\right\}=\left\{\begin{array}{c}
556.7703 \\
613.3457 \\
-1.4557 \\
45.1338
\end{array}\right\} \text { y } a_{1}=-40.6282 \quad \text { Grupo } 1 \\
& \Rightarrow\left\{\begin{array}{l}
b_{1,2} \\
b_{2,2} \\
b_{3,2} \\
b_{4,2}
\end{array}\right\}=\left\{\begin{array}{r}
975.7229 \\
1242.6354 \\
-3.2838 \\
82.2430
\end{array}\right\} \text { y } a_{2}=-139.4446 \quad \text { Grupo } 2
\end{aligned}
$$

Así, las Funciones Discriminantes Lineales de Fisher para los Grupos 1 y 2 son, respectivamente:

$$
\begin{aligned}
& F_{1}=556.770 X_{9}+613.346 X_{6}-1.456 X_{11}+45.134 X_{5}-40.628 \\
& F_{2}=975.723 X_{9}+1242.635 X_{6}-3.284 X_{11}+82.243 X_{5}-139.445
\end{aligned}
$$

Para ambos grupos, los coeficientes y la constante son:

$$
\left\{\begin{array}{l}
b_{1} \\
b_{2} \\
b_{3} \\
b_{4}
\end{array}\right\}=\left\{\begin{array}{l}
b_{1,2} \\
b_{2,2} \\
b_{3,2} \\
b_{4,2}
\end{array}\right\}-\left\{\begin{array}{l}
b_{1,1} \\
b_{2,1} \\
b_{3,1} \\
b_{4,1}
\end{array}\right\}=\left\{\begin{array}{c}
418.953 \\
629.290 \\
-1.828 \\
37.109
\end{array}\right\} \text { y } a=a_{2}-a_{1}=-98.816
$$

Por tanto, la Función Discriminante de Clasificación Lineal de Fisher, para ambos grupos, es:

$$
D-C=418.953 X_{9}+629.290 X_{6}-1.828 X_{11}+37.109 X_{5}-98.816
$$

La tabla 11 muestra la clasificación de los bancos en estudio empleando esta última función. 
Tabla 11

Clasificación de los Bancos en estudio utilizando la Función Discriminante Lineal de Fisher

\begin{tabular}{cccccccc}
\hline Banco & $\begin{array}{c}\text { Grupo de } \\
\text { pertenencia }\end{array}$ & $\begin{array}{c}\text { Rentabilidad sobre } \\
\text { activos productivos }\end{array}$ & Morosidad & Eficiencia & $\begin{array}{c}\text { Liquidez } \\
\text { (MACRO) }\end{array}$ & $\begin{array}{c}\text { Puntuación } \\
\text { discriminante }\end{array}$ & $\begin{array}{c}\text { Clasificado } \\
\text { como }\end{array}$ \\
\hline 1 & 1 & 0.027 & 0.064 & 1.734 & 0.531 & -30.558 & 1 \\
2 & 1 & 0.059 & 0.048 & 0.866 & 0.503 & -26.936 & 1 \\
3 & 1 & 0.002 & 0.175 & 36.475 & 0.581 & -32.991 & 1 \\
4 & 1 & 0.074 & 0.040 & 1.037 & 0.429 & -28.778 & 1 \\
5 & 1 & 0.058 & 0.013 & 0.927 & 0.796 & -38.373 & 1 \\
6 & 2 & 0.084 & 0.087 & 0.864 & 1.121 & 31.376 & 2 \\
7 & 2 & 0.078 & 0.103 & 0.859 & 0.520 & 16.027 & 2 \\
8 & 2 & 0.094 & 0.117 & 0.638 & 0.712 & 39.437 & 2 \\
9 & 2 & 0.086 & 0.115 & 1.052 & 0.840 & 39.269 & 2 \\
\hline
\end{tabular}

Fuente: Elaboración propia

En lo referente a las probabilidades de pertenencia a los grupos, éstas se obtuvieron con:

$$
\operatorname{Prob}(g / D)=\frac{\pi_{g} e^{F_{g}}}{\pi_{1} e^{F_{1}}+\pi_{2} e^{F_{2}}} \quad g=1,2
$$

Donde $\pi_{g}$ y $F_{g}$ son la probabilidad a priori y la Función Discriminante lineal de Fisher, respectivamente, del $g$-ésimo grupo.

En la tabla 12 se muestran las probabilidades de pertenencia a los Grupos 1 y 2 de acuerdo con las Funciones Discriminantes Lineales de Fisher y las probabilidades a priori.

Tabla 12

Probabilidades de pertenencia a los grupos de Bancos en dificultades y Bancos no en dificultades

\begin{tabular}{ccccccccc}
\hline Banco & $\begin{array}{c}\text { Rentabilidad sobre } \\
\text { activos productivos }\end{array}$ & Morosidad Eficiencia & $\begin{array}{c}\text { Liquidez } \\
\text { (MACRO) }\end{array}$ & $F_{1}$ & $F_{2}$ & Prob(2/D) & Prob(1/D) \\
\hline 1 & 0.027 & 0.064 & 1.734 & 0.531 & 35.174 & 4.616 & 0.0000 & 1.0000 \\
2 & 0.059 & 0.048 & 0.866 & 0.503 & 42.925 & 15.989 & 0.0000 & 1.0000 \\
3 & 0.002 & 0.175 & 36.475 & 0.581 & 40.951 & 7.960 & 0.0000 & 1.0000 \\
4 & 0.074 & 0.040 & 1.037 & 0.429 & 42.842 & 14.064 & 0.0000 & 1.0000 \\
5 & 0.058 & 0.013 & 0.927 & 0.796 & 34.317 & -4.055 & 0.0000 & 1.0000 \\
6 & 0.084 & 0.087 & 0.864 & 1.121 & 109.046 & 140.421 & 1.0000 & 0.0000 \\
7 & 0.078 & 0.103 & 0.859 & 0.520 & 87.797 & 103.823 & 1.0000 & 0.0000 \\
8 & 0.094 & 0.117 & 0.638 & 0.712 & 114.628 & 154.065 & 1.0000 & 0.0000 \\
9 & 0.086 & 0.115 & 1.052 & 0.840 & 114.654 & 153.924 & 1.0000 & 0.0000 \\
\hline
\end{tabular}

Fuente: Elaboración propia. 
Como puede observarse en las Tablas 11 y 12, el modelo propuesto identifica perfectamente la clasificación de los nueve bancos mexicanos que estuvieron en la crisis bancaria mexicana.

Aplicación Predictiva de la Situación Financiera de Cuatro Bancos Mexicanos y Uno estadounidense.

Con la Función Discriminante de Clasificación Lineal de Fisher (ec. 21) se predijo la situación de los bancos mexicanos 1, 5, 6 y 7, la cual se muestra en la tabla 13. Los resultados empíricos de la predicción concuerdan, en general, con los análisis reportados por el IPAB 1999 - 2005 (véase lo expuesto en la tabla 1) y BANXICO en sus informes anuales para el periodo 2007-2008; nótese la recuperación de los bancos en el 2008, lo que también concuerda con lo expuesto en el Inciso II. La misma predicción se realizó para un banco de los Estados Unidos, resultando lo mostrado en la tabla 14. Como puede observarse, el resultado predicho no concuerda con los informes anuales reportados por los Presidentes de dicho banco para el periodo 2000 - 2006 pero sí para el 2007 - 2010. En relación con este último periodo, es necesario señalar que el modelo propuesto presenta una recuperación en los años 2009 y 2010 reflejada por la tendencia hacia valores positivos de la función mencionada. Esto concuerda con el hecho de que este banco se adhirió al Programa de Alivio de Activos (TARP, por sus siglas en inglés), el cual fue aprobado por el Congreso de Estados Unidos en octubre del 2008 para la restauración de los mecanismos de formación de precios en los mercados mediante la compra de activos con problemas de valoración por el Departamento del Tesoro de dicho país (Calvo y Martín de Vidales, 2014). Es necesario comentar que Cools y van Toor, 2010, consideran a Bank of America como un banco débil (en dificultades) durante el periodo 2002-2006.

Tabla 13

Predicción de cuatro bancos para saber si está o no en dificultades

\begin{tabular}{|c|c|c|c|c|c|c|c|c|}
\hline \multirow{2}{*}{$\begin{array}{l}\text { Año } \\
2000\end{array}$} & \multicolumn{2}{|c|}{ Serfín } & \multicolumn{2}{|c|}{ Santander Mexicano } & \multicolumn{2}{|c|}{ Banorte } & \multicolumn{2}{|c|}{ BBVA Bancomer ${ }^{(* * *)}$} \\
\hline & -57.232 & En Dific. & -36.679 & En Dific. & -30.195 & En Dific. & -3.694 & En Dific. \\
\hline 2001 & -40.947 & En Dific. & -23.286 & En Dific. & -29.856 & En Dific. & -16.414 & En Dific. \\
\hline 2002 & -45.756 & En Dific. & -52.683 & En Dific. & -43.455 & En Dific. & -25.149 & En Dific. \\
\hline 2003 & -29.311 & En Dific. & -32.917 & En Dific. & -49.125 & En Dific. & -33.464 & En Dific. \\
\hline 2004 & -36.993 & En Dific. & -24.717 & En Dific. & -42.474 & En Dific. & -38.618 & En Dific. \\
\hline 2005 & $-19.201^{(* *)}$ & En Dific. & $-20.500^{(* *)}$ & En Dific. & -34.014 & En Dific. & -38.136 & En Dific. \\
\hline 2006 & $-31.153^{(*)}$ & En Dific. & $-31.153^{(*)}$ & En Dific. & -36.168 & En Dific. & -29.894 & En Dific. \\
\hline 2007 & $-21.343^{(*)}$ & En Dific. & $-21.343^{(*)}$ & En Dific. & -42.058 & En Dific. & -32.134 & En Dific. \\
\hline 2008 & $24.052^{(*)}$ & No en Dific. & $24.052^{(*)}$ & No en Dific. & 6.197 & No en Dific. & -2.744 & En Dific. \\
\hline
\end{tabular}

(*) Banca Serfín se fusionó a Banco Santander Mexicano a partir de diciembre de 2004

(**) En 2005 tenía valores promedio diferentes

(***) Bancomer se fusionó a BBVA - Probursa a partir de agosto de 2000

Fuente: Elaboración propia 
Tabla 14

Predicción de un banco de los Estados Unidos para saber si está ó no en dificultades

\begin{tabular}{lll}
\hline Año & \multicolumn{2}{c}{ Bank of America } \\
\hline 2000 & -67.917 & En Dific. \\
2001 & -63.764 & En Dific. \\
2002 & -63.788 & En Dific. \\
2003 & -68.365 & En Dific. \\
2004 & -67.829 & En Dific. \\
2005 & -70.852 & En Dific. \\
2006 & -73.656 & En Dific. \\
2007 & -73.820 & En Dific. \\
2008 & -63.542 & En Dific. \\
2009 & -46.257 & En Dific. \\
2010 & -48.772 & En Dific. \\
\hline
\end{tabular}

Fuente: Elaboración propia

Por lo anterior, se puede mencionar la bondad del modelo propuesto en el sentido de la predicción. Además, aun cuando el IPAB determinó que las instituciones aquí analizadas, en relación con los niveles de capitalización, cumplían con lo solicitado en el Nuevo Programa del PCCC en el periodo 2000-2003 y, según la CNBV, las mismas se clasificaban en la categoría I a partir del 2004 a 2008, cabe señalar que la crisis financiera internacional suscitó diversos cuestionamientos a las reglas de capitalización alineadas con las recomendaciones del BCBS. En particular, se ha dudado de la bondad del índice de capitalización como indicador de la solvencia bancaria (BANXICO, Reporte sobre el Sistema Financiero 2009). Por otra parte, el total de las provisiones, calculado como proporción de la cartera vencida de los bancos Banorte, Banamex, Santander, BBVA Bancomer, y HSBC, permaneció estable en el 2006. Sin embargo, en el 2007, estos bancos redujeron sus utilidades debido a la disminución de los ingresos por intermediación y a los mayores egresos por creación de provisiones. Posteriormente, en el 2008, las provisiones se vieron afectadas por un incremento sustancial de los créditos con calificación menor a "A" de la banca múltiple derivado de un aumento en la morosidad (BANXICO, Reporte sobre el Sistema Financiero 2009). De esta manera, se puede observar que, si bien la insuficiencia de capital se previene tomando en cuenta el índice de capitalización, la insuficiencia de reservas preventivas se determina a través del nivel de créditos de los bancos.

Aun cuando las crisis financieras no son la única fuente de desaceleración de la economía, cuantificada por el Producto Interno Bruto (PIB), la turbulencia de los mercados financieros internacionales afectaron significativamente el desempeño de la economía mundial durante, 
y después, del año 2008 (BANXICO, Informe anual 2008). En particular, dado que en este trabajo se analizan de manera predictiva en su situación financiera cuatro bancos mexicanos y uno estadounidense, en la Tabla 15 se presenta la variación anual del PIB (en por ciento) de México y Estados Unidos en el periodo 2006 - 2010. Como puede observarse en dicha Tabla, el crecimiento de la economía fue muy bajo en dicho periodo, lo cual concuerda con las predicciones presentadas en las Tablas 13 y 14.

Tabla 15

Variación anual del Producto Interno Bruto (PIB) en por ciento(*)

\begin{tabular}{cccccc}
\hline País & 2006 & 2007 & 2008 & 2009 & 2010 \\
\hline México & 4.9 & 3.3 & 1.5 & -6.5 & 5.5 \\
Estados Unidos & 2.7 & 2.1 & 0.4 & -2.4 & 2.9 \\
\hline
\end{tabular}

(*) Fuente BANXICO, Informe anual, 2009 y 2010

Fuente: Elaboración propia

\section{Conclusiones}

Hemos presentado un modelo de análisis discriminante para identificar y predecir la situación financiera de las instituciones de banca múltiple mediante una combinación de indicadores financieros pertenecientes a diferentes metodologías que muestran un enfoque eminentemente predictivo. En lo que a la identificación se refiere, se observa que este análisis tiene una aplicación explicativa veraz como lo muestra la función discriminante obtenida, la cual es significativa con un alto poder discriminante ya que el valor de la correlación canónica fue de $0.976 \mathrm{y}$, por su parte, las probabilidades de pertenencia a uno u otro grupo (tabla 11) fueron 1. En relación con la predicción, también se mostró que la aplicación predictiva a los cuatro bancos mexicanos analizados en el periodo 2000-2008, y a uno estadounidense analizado en el periodo 2000-2010, es confiable ya que generalmente concuerda con los análisis emitidos por el IPAB, BANXICO y los presidentes del Bank of America. Ambas aplicaciones se realizaron con base en las variables clasificadoras resultantes del método paso a paso como lo fueron la Rentabilidad sobre Activos Productivos, Morosidad, Eficiencia y Liquidez, (MACRO), los cuales están estrechamente relacionados con la crisis financiera nacional y mundial. En el caso de la Rentabilidad sobre Activos Productivos, el cual contempla las operaciones con instrumentos financieros derivados, se puede señalar que, aún cuando éste fue seleccionado con datos del año de 1999 mediante el modelo propuesto, todavía juega un papel importante en lo que a crisis financieras se refiere como se constata con lo expuesto en la Ley Dodd-Frank (Dodd-Frank Wall Street Reform) en Estados Unidos (González y Marqués, 2010) y la EMIR 
(European Market Infrastructure Regulation) en Europa (Reglamento del Parlamento Europeo y del Consejo, 2012), donde se manifiesta que el común denominador de estas iniciativas es que se enfocan principalmente en los derivados, los cuales contribuyeron a la desestabilización de los mercados (Asobancaria, 2013). Por otra parte, estos productos financieros citados, además de la estructuración y proliferación de otros créditos de baja calidad así como la incidencia de la política económica en general dentro de la economía real -periodo 2004-2011 - y sobre el desempeño de aquéllos, se pueden citar en el caso de España, donde su consecuencia inmediata fue el deterioro de la liquidez y del financiamiento llevándola a una recesión a finales de 2008 (Marín, et al., 2015). En relación con el margen bruto financiero, éste se ve influenciado por las variaciones del tipo de interés que afectan directamente a los flujos de costos y de ingresos de las entidades bancarias (Ballester, et. al., 2009).

Con respecto a la morosidad, Ana Fernández-Sainz y Felipe Llaugel en 2011 recalcan que algunas variables relativas al sector bancario ayudaron a explicar la posibilidad de problemas, aunque no el momento de la crisis. Entre las variables específicas consideradas estuvieron: la razón de capital ajustada por riesgo, la cartera vencida entre total de cartera y la concentración del crédito como proporción de la cartera total.

Los anteriores autores también manifiestan que numerosos estudios han demostrado que el análisis de los indicadores, de forma individual, puede ser engañoso, en especial cuando lo que se busca es determinar un umbral dentro del cual cada indicador debe estar ubicado y que, en cuanto sobrepase dicho umbral, se genere una señal de alerta. Así, esto implica el desarrollo de una metodología que analice, no los indicadores de forma individual, sino al patrón que muestra el conjunto de indicadores de relevancia. Asimismo, se puede decir que diversas investigaciones fortalecen la hipótesis de que no son los indicadores individuales los que deben ser analizados, sino el patrón de comportamiento que presentan los mismos. En adición, dichos autores mencionan que algunos trabajos (Todorov et al., 1999) indican que la función de clasificación (técnica estadística de análisis multivariante) se puede construir con un número relativamente reducido de indicadores, debido a la gran correlación que comúnmente se observa entre ellos.

En relación con los indicadores financieros empleados en este trabajo de investigación, es necesario señalar que Altman, et al., 2014, en su artículo “Anatomía de Bancos en Crisis”, contempla indicadores financieros pertenecientes al sistema CAMEL similares o iguales a los aquí analizados, provenientes de la base de datos Bankscope. No existe una teoría general que guíe el proceso de selección de dichos indicadores (Labatut, et. al., 2009). Sin embargo, el contexto de cada uno de los trece indicadores que se han considerado en este trabajo está o estará contemplado, en menor o mayor relevancia, en los acuerdos de Basilea II y III del BCBS a partir del año 2008 como se muestra en la tabla 5, lo cual realza la relevancia de la selección de cada uno y la vigencia del empleo de los indicadores que aquí se utilizaron. 
Sin duda, la situación presente y posible evolución futura económica de un país o área mundial se refleja, normalmente en primera instancia, en la economía financiera y ésta donde primero se muestra es a través de la información financiera de los Bancos. Por ello resulta de gran interés disponer de metodologías, verificadas en el terreno de la praxis, que nos aporten información veraz y en tiempo de esta posible evolución y que además controle variables claves en banca como son la solvencia vía recursos propios así como los adecuados registros de posibles moras vía provisiones crediticias. Tras la revisión bibliográfica realizada estamos en condiciones de afirmar que la metodología que proponemos está en la línea de las últimas tendencias en análisis ex ante de predicción de solvencia bancaria que son analizadas y sustentadas por el BIS, Comité de Basilea, Test de Strees de la Banca Europea así como el análisis nacional, entre otros. Este análisis e información permitiría desarrollar una política económica y financiera nacional y/o supranacional con el debido tiempo que atenuará los efectos sobre la economía real y su incidencia en todas las variables clave para el desarrollo económico.

Por lo anterior, se concluye que el modelo propuesto puede ser utilizado, adicionalmente a los modelos de alerta temprana institucionales, para predecir si un banco está o no en dificultades. Al respecto, es necesario mencionar que dicho modelo, aun cuando no toma en cuenta el ICAP tal como lo hacen los modelos de alerta temprana, sí considera la provisión para pérdidas crediticias. Adicionalmente, este modelo puede ser aplicado a cualquier banco de cualquier país y en cualquier tiempo posterior a los años analizados en este trabajo, considerando que, para su aplicación, deberá contarse con la información financiera actualizada necesaria, e importante en el sector bancario (Gras, et. al., 2015), para el cálculo de los indicadores financieros propuestos en este trabajo.

\section{Referencias}

Alfaro, E., Gámez, M. y García, N. (2008). Linear discriminant analysis versus adaboost for failure forecasting. Revista Española de Financiación y Contabilidad, 37(137), 13-32. Disponible en: https://www.aeca.es/old/ refc_1972-2013/2008/137-2.pdf y consultado: 20/10/2009.

Alkhatib, K. \& Eqab, A. (2011). Predicting Corporate Bankruptcy of Jordanian Listed Companies: Using Altman and Kida Models. International Journal of Business and Management 6(3). https://doi.org/10.5539/ijbm.v6n3p208.

Altman, E. I. (1968). Financial Ratios, Discriminant Analysis and the Prediction of Corporate Bankruptcy. The Journal of Finance, 23(4), 589-609. https://doi.org/10.2307/2978933.

Banco de México (2006). Informe Anual. Editado por el Banco de México. Disponible en: http://www.banxico.org. $\mathrm{mx} /$ dyn/publicaciones-y-discursos/publicaciones/informesperiodicos/anual/\%7B4EF1BFA3-67A1-F187-0DB 0-1243591806B5\%7D.pdf y consultado: 09/11/2014.

Banco de México (2007). Informe Anual. Editado por el Banco de México. Disponible en: http://www. banxico.org.mx/dyn/publicaciones-y-discursos/publicaciones/informesperiodicos/anual/\%7BE1AEF-

D7D-BD92-25FB-1733-D1ABC6467518\%7D.pdf y consultado: 18/02 2011.

Banco de México (2008). Informe Anual. Editado por el Banco de México. Disponible en: http://www.banxico.org. mx/publicaciones-y-discursos/publicaciones/informesperiodicos/anual/\%7BE2479C99-47CB-19B8-92A7-

D011876E8FCA\%7D.pdf y consultado: 18/02/2011. 
Banco de México (2006). Reporte sobre el Sistema Financiero. Editado por el Banco de México. Disponible en: http://www.banxico.org.mx/dyn/publicaciones-y-discursos/publicaciones/informes-periodicos/reporte-sf/\%7B1507E4EF-CC92-7236-34E2-FA08BD0FA668\%7D.pdf y consultado: 15/10/2013.

Banco de México (2007). Reporte sobre el Sistema Financiero. Editado por el Banco de México. Disponible en: http://www.banxico.org.mx/dyn/publicaciones-y-discursos/publicaciones/informes-periodicos/reporte-sf/\%7BE3230E6A-48B0-04D0-A58C-FB92F38B2EC9\%7D.pdf y consultado: 03/04/2011.

Banco de México (2009). Reporte sobre el Sistema Financiero. Editado por el Banco de México. Disponible en: http://www.banxico.org.mx/dyn/publicaciones-y-discursos/publicaciones/informes-periodicos/reportesf/\%7B5286741D-A39E-9745-B393-AF3DF0A5AE85\%7D.pdf y consultado: 29/06/2017.

Ballester, L., Ferrer, R. y González C. (2009). Impacto del riesgo de interés sobre las acciones del sector bancario español. Spanish Journal of Finance and Accounting / Revista Española De Financiación y Contabilidad. 38(142), 213-238. https://doi.org/10.1080/02102412.2009.10779667.

Beaver, W. H. (1966). Financial Ratios as Predictors of Failure. Journal of Accounting Research, 4, 71-111. https:// doi.org/10.2307/2490171 (1968). Market Prices, Financial Ratios, and the Prediction of Failure. Journal of Accounting Research, 6(2), 179-192. https://doi.org/10.2307/2490233.

Calvo, A. y Martín de Vidales, I. (2014). El Rescate Bancario: Importancia y Efectos Sobre Algunos Sistemas Financieros Afectados. Revista de Economía Mundial, núm. 37, 125-150. Disponible en: http://www.redalyc.org/ pdf/866/86632964006.pdf y consultado: 10/08/2017.

Carrasco, A. (1999). Fundamentos de los sistemas de alerta en las entidades de supervisión bancaria. Revista Española de Financiación y Contabilidad, 28(102), 1043-1074. Disponible en: https://dialnet.unirioja.es/servlet/ articulo? codigo $=44319$ y consultado: $12 / 08 / 2009$.

Chávez, N., Córdova, C. y Alvarado, P. (2017). Medición del riesgo de la gestión financiera de las compañías con la utilización del análisis discriminante: el caso de las industrias de la región 7 del Ecuador. Revista Publicando, 4 No 13. No.Esp. UTPL. 2017,90-107.ISSN 1390-9304. Disponible en: https://www.rmlconsultores.com/revista/ index.php/crv/article/view/779 y consultado: 09/01/2018.

Climent, S. (2017) Dotaciones para los deterioros de los créditos. Un estudio por ciclos económicos. Cuadernos de Economía. 40(112), 56-67. https://doi.org/10.1016/j.cesjef.2016.01.001.

Correa, E. (1999). Crisis y rescate bancario México. Revista Pueblos, Alianza Unida, España. Disponible en: http:// www.redcelsofurtado.edu.mx/archivosPDF/correa5.pdf y consultado: 05/06/2017.

Crespo, J. Y. (2011). CAMEL vs. discriminante, un análisis de riesgo al sistema financiero venezolano. Ecos de Economía, ISSN 1657-4206, Año 15, No. 33, 25-47. Disponible en: https://dialnet.unirioja.es/descarga/articulo/3974842.pdf y consultado: 19/02/2017.

El-Ansary, O. y Hafez, H. (2015). Determinants of Capital Adequacy Ratio: an empirical study on Egyptian Banks. Corporate Ownership and Control. 13(1) https://doi.org/10.22495/cocv13i1c10p4.

Fernández-Sainz, A. y Llaugel F. (2011). ¿Bancos con Problemas? Un Sistema de Alerta Temprana para la Prevención de Crisis Bancarias. Revista Cuadernos de Gestión 11(2), 149-168. https://doi. org/10.5295/cdg.100239af.

Gamlath, G. R. M. y Rathiranee, Y. (2014). Effects of Working Capital Management and Profitability: Evidence from Listed Commercial Banks in Sri Lanka. Journal of Business Studies. Disponible en: https://papers.ssrn.com/sol3/papers.cfm?abstract_id=2444294 y consultado: 06/05/2017.

Gras, E., Marín, S. y García, D. (2015). Auditoría interna y deficiencias de la información financiera en el sector bancario español. Revista de Contabilidad 18(2), 174-181. https://doi.org/10.1016/j. rcsar.2014.06.004.

Gutiérrez, C. y Abad, J. (2014). ¿Permitían los estados financieros predecir los resultados de los tests de estrés de la banca española? Una aplicación del modelo logit. Revista de Contabilidad. 17(1), 58-70. https://doi.org/10.1016/j.rcsar.2013.08.004. 
Hazera, A., Quirvan, C. y Marín, S. (2016). The impact of guaranteed bailout assistance on bank loan overstatement. International Journal of Managerial Finance. 12(2), 177-210. https://doi. org/10.1108/ijmf-04-2014-0046.

Hernández, F. y López O. (2001). La crisis bancaria mexicana: Un modelo de duración y riesgo proporcional. El trimestre económico 272, 551-601. Disponible en: https://media.proquest.com/ media/pq/classic/doc/870986801/fmt/pi/rep/NONE?_s=hQBKSeB4pNKd\%2FvdciBA3Jxuu8sQ\%3D y consultado: 19/10/2009.

IPAB (1999). Informe Anual. Editado por el IPAB. Disponible en: http://www.gob.mx/cms/uploads/ attachment/file/101165/Informe-Anual_1999.pdf y consultado: 08/11/2011.

IPAB (2000). Informe Anual. Editado por el IPAB. Disponible en: http://www.gob.mx/cms/uploads/ attachment/file/101166/Informe-Anual_2000.pdf y consultado: 28/07/2011.

IPAB (2001). Informe Anual. Editado por el IPAB. Disponible en: http://www.gob.mx/cms/uploads/ attachment/file/101167/Informe-Anual_2001.pdf y consultado: 05/02/2012.

IPAB (2002). Informe Anual. Editado por el IPAB. Disponible en: http://www.gob.mx/cms/uploads/ attachment/file/101168/Informe-Anual_2002.pdf y consultado: 05/02/2012.

IPAB (2003). Informe Anual. Editado por el IPAB. Disponible en: http://www.gob.mx/cms/uploads/ attachment/file/101170/Informe-Anual_2003.pdf y consultado: 05/02/2012.

IPAB (2005). Informe Anual. Editado por el IPAB. Disponible en: http://www.gob.mx/cms/uploads/ attachment/file/101171/Informe-Anual_2005.pdf y consultado: 04/04/2011.

Labatut, G., Pozuelo, J. y Veres, E. J. (2009). Modelización temporal de los ratios contables en la detección del fracaso empresarial de la PYME española. Spanish Journal of Finance and Accounting / Revista Española de Financiación y Contabilidad. 38(143), 423-447. https://doi.org/10.1080/02 102412.2009.10779672.

Laffarga, J., Martín, J. L. y Vázquez, M. J. (1987). Predicción de la crisis bancaria en España: comparación entre el análisis logit y el análisis discriminante. Cuadernos de Ciencias Económicas y Empresariales, $\mathrm{N}^{\circ}$ 18, 49-57. (1991). Disponible en: https://idus.us.es/xmlui/bitstream/handle/11441/78703/Predicci\%C3\%B3n\%20de\%20la\%20crisis\%20bancaria\%20en\%20Espa\%C3\%B1a.pdf?sequence=1 y consultado: 22/08/2009.

Laffarga, J., Martín, J. L. y Vázquez, M. J. (1991). La predicción de la quiebra bancaria el caso español. Revista Española de Financiación y Contabilidad, 21(66), 151-166. Disponible en: https:// dialnet.unirioja.es/descarga/articulo/44024.pdf y consultado: 22/08/2009.

Lifschutz, S. (2010).Predicting Bankruptcy: Evidence from Israel. International Journal of Business and Management. 5(4). https://doi.org/10.5539/ijbm.v5n4p133.

López, G. y P. N. Snowden (1999). La banca mexicana, de la privatización a la intervención Una perspectiva del AED, 1982-1996, El Trimestre Económico, 66 (262(2)), 259-291. Disponible en: https://www.jstor.org/stable/20856987?seq=1\#page_scan_tab_contents y consultado: 19/10/2009.

Marín, S., Gras, E. y Anton, M. (2015). Spanish credit institutions: do efficiency and solvency support the first decisions on bank restructuring from a financial point of view 2008-2012?. Revista de Contaduría y Administración, UNAM, 60(2), 367-388. https://doi.org/10.1016/s0186-1042(15)30005-x.

Marín, S., Mondragón, Z. y Anton, M. (2011). Crisis bancarias, información financiera y modelos de predicción. Estudio de un caso. Revista de Globalización, Competitividad y Gobernabilidad. ISSN 1988-7116, 5(1), Enero-Abril 2011, 32-41. https://doi.org/10.3232/GCG.2011.V5.N1.02.

Naresh, M. \& Sree, V. (2014). A New Methodology for Estimating Internal Credit Risk and Bankruptcy Prediction under Basel II Regime. Computational Economics. 46(1), 83-102. https://doi. org/10.1007/s10614-014-9452-9.

Mora, A. (1994). Limitaciones metodológicas de los trabajos empíricos sobre la predicción del fracaso empresarial. Revista Española de Financiación y Contabilidad, 24(80), 709-732. Disponible en: https://dialnet.unirioja.es/descarga/articulo/44141.pdf y consultado: 13/08/2009. 
Ríos, H. y Gómez, T. (2015). Competencia, Eficiencia y Estabilidad Financiera en el Sector Bancario Mexicano. Revista Mexicana de Economía Y Finanzas. 10(1), 3958. https://doi.org/10.21919/remef.v10i1.65.

\section{Libros}

Altman, E. I. (2000). Predicting Financial Distress of Companies: Revisiting the Z-Score and Zeta® Models. Handbook of Research Methods and Applications in Empirical Finance. 428-456. https:// doi.org/10.4337/9780857936097.00027.

Burden, R. y Faires D. (1985). Análisis Numérico. Grupo Editorial Iberoamérica.

Ferrán, M. (2001). SPSS PARA WINDOWS. Análisis Estadístico. McGRAW-HILL/INTERAMERICANA DE ESPAÑA, S. A. U.

Hair, J. Jr., R. Anderson, R. Tatham, y W. Black (2005). Análisis Multivariante. 5a. edición, PEARSON, Prentice Hall.

Marín, S. y F. J. Martínez (2002). Contabilidad Bancaria en México. Instituto Mexicano de Contadores Públicos A. C. y Universidad de Cantabria.

Pérez, C. (2005). Métodos Estadísticos Avanzados con SPSS. Instituto de Estudios Fiscales, Universidad Complutense, Thomson Editores España.

Uriel, E. y J. Aldás (2005). Análisis Multivariante Aplicado, Thomson Editores Spain.

\section{Versiones electrónicas}

Acuña, E. (2000). Notas de Análisis Discriminante. Disponible en: Disponible en: http://profesores.usfq.edu.ec/fdelgado/Teor\%C3\%ADa\%20y\%20M\%C3\%A9todos\%20en\%20Arqueolog\%C3\%ADa/Archivos\%20Digitales/metodos/clasifall.pdf y consultado: 20/04/2010.

Altman, E. I., Cizel, J. y Rijken, H. A. (2014). Anatomy of Bank Distress: The Information Content of Accounting Fundamentals Within and Across Countries. https://doi.org/10.2139/ssrn.2504926.

Amieva, J. y B. Urriza (2000). Crisis Bancarias: causas, costos, duración, efectos y opciones de política. CEPAL División Desarrollo Económico. Disponible en: http://archivo.cepal.org/pdfs/2000/ S00135.pdf y consultado: 01/10/2014.

Anastasi, A., T. Burdisso, E. Grubisic y S. Lencioni (1998). ¿Es posible anticipar problemas en una entidad financiera? Argentina 1994-1997. Disponible en: http://cdi.mecon.gov.ar/biblio/docelec/ aaep/98/anastasi_burdisso_grubisic.pdf y consultado: 09/12/2008.

Arango, C. y L. Botero (2001). Evaluación del Modelo CAMEL como Instrumento de Prevención de Crisis Bancarias. Disponible en: http://www.eafit.edu.co/NR/rdonlyres/AEA11F52-A6B0-4DC6AF5E-801539998C28/0/Evaluaci\%C3\%B3nDelModeloCameltrabajodegrado.pdf y consultado: $14 / 03 / 2010$.

Asobancaria (2013). Efectos extraterritoriales de la Ley Dodd-Frank. Disponible en http://www.asobancaria.com/portal/pls/portal/docs/1/4392639.PDF y consultado: 27/01/2015.

Ayala, R. (1999). Modelos de Alerta Temprana para Crisis Financieras. El Caso Ecuatoriano: 19941997. Disponible en: http://www.bce.fin.ec/docs.php?path=./documentos/Publicaciones Notas/ Catalogo/NotasTecnicas/nota51.pdf y consultado: 21/05/2009.

Comisión Nacional del Mercado de Valores (2012). Reglamento del Parlamento Europeo y del Consejo, 2012 relativo a los derivados extrabursátiles, las entidades de contraparte central (ECCs) y los registros de operaciones (en adelante, EMIR). Disponible en https://www.cnmv.es/portal/GPage. aspx ?id=EMIR_ReqOblig\&idpf=6 y consultado: 04/03/2015.

Comité de Supervisión Bancaria de Basilea (2002). Orientaciones para la Supervisión de Bancos en Dificultades. Banco de Pagos Internacionales, marzo. Disponible en: http://www.bis.org/publ/bcbs88esp.pdf y consultado: 21/03/2011. 


\section{M. Gutiérrez Ramírez, et al./ Contaduría y Administración 64(4), 2019, 1-37}

http://dx.doi.org/10.22201/fca.24488410e.2018.1770

Comité de Supervisión Bancaria de Basilea (2006). Convergencia internacional de medidas y normas de capital. Junio. Disponible en: http://www.bis.org/publ/bcbs128_es.pdf y consultado: 08/10/2016.

Comité de Supervisión Bancaria de Basilea (2008). Principios para la adecuada gestión y supervisión del riesgo de liquidez. Banco de Pagos Internacionales, septiembre. Disponible en: http://www.bis.org/publ/bcbs144_es.pdf y consultado: 25/06/2016.

Comité de Supervisión Bancaria de Basilea (2010). La respuesta del Comité de Basilea a la crisis financiera: informe al G-20. Banco de Pagos Internacionales, octubre. Disponible en: http://www.bis.org/publ/bcbs179_es.pdf y consultado: 04/10/2012.

Comité de Supervisión Bancaria de Basilea (2010) Basilea III. Marco internacional para la medición, normalización y seguimiento del riesgo de liquidez, diciembre. Disponible en: http://www.bis.org/publ/bcbs188_es.pdf y consultado: 14/06/2016.

Comité de Supervisión Bancaria de Basilea (2011). Marco regulador global para reforzar los bancos y sistemas bancarios, revisado en junio. Disponible en: http://www.bis.org/publ/bcbs189_es.pdf y consultado: 03/09/2012.

Comité de Supervisión Bancaria de Basilea (2013). Coeficiente de cobertura de liquidez (LCR) y herramientas de seguimiento del riesgo de liquidez, enero. Disponible en: http://www.bis.org/publ/bcbs238_es.pdf y consultado: 27/05/2016.

Comité de Supervisión Bancaria de Basilea (2013). El marco regulador: equilibrio entre sensibilidad al riesgo, sencillez y comparabilidad, julio. Disponible en: http://www.bis.org/publ/bcbs258_es.pdf y consultado: 24/11/2016.

Comité de Supervisión Bancaria de Basilea (2014). Coeficiente de Financiación Estable Neta (NSFR), octubre. Disponible en: http://www.bis.org/bcbs/publ/d295_es.pdf y consultado: 28/05/2016.

Comité de Supervisión Bancaria de Basilea (2015). Orientaciones sobre la contabilidad de pérdidas crediticias esperadas, febrero. Disponible en: http://www.bis.org/bcbs/publ/d311_es.pdf y consultado: 09/11/2016.

Comité de Supervisión Bancaria de Basilea (2015). Orientaciones para identificar y hacer frente a bancos en dificultades. Banco de Pagos Internacionales, julio. Disponible en: https://www.bis.org/bcbs/publ/d330_es.pdf y consultado: 19/10/2018.

Comité de Supervisión Bancaria de Basilea (2015). Orientaciones sobre riesgo de crédito y contabilidad de pérdidas crediticias esperadas, diciembre. Disponible en: http://www.bis.org/bcbs/publ/d350_es.pdf y consultado: 09/11/2016.

Comité de Supervisión Bancaria de Basilea (2016). Revisión del marco del coeficiente de apalancamiento, abril. Disponible en: http://www.bis.org/bcbs/publ/d365_es.pdf y consultado: 27/05/2016.

Comité de Supervisión Bancaria de Basilea (2016). Riesgo de tasas de interés en la cartera de inversión, abril. Disponible en: http://www.bis.org/bcbs/publ/d368_es.pdf y consultado: 30/05/2016.

Cools, K. y van Toor, J. (2015). Why Did US Banks Fail? What Went Wrong at US Banks in the Run Up to the Financial Crisis. SSRN Electronic Journal. https://doi.org/10.2139/ssrn.2566737.

González, B. H. (2006). La prueba de Shapiro \& Wilk para verificar la normalidad de un conjunto de datos proveniente de muestras pequeñas. Universidad Rafael Landívar. Disponible en: https://es.scribd.com/doc/266965243/ Verificacion-de-Normalidad-Shapiro-y-Wilk y consultado: 10/05/2009.

González y Marqués (2010). Dodd-Frank Wall Street Reform: un cambio profundo en el sistema financiero de Estados Unidos. Dirección General Adjunta de Asuntos Internacionales del Banco de España. Disponible en: http:// www.bde.es/f/webbde/Secciones/Publicaciones/InformesBoletinesRevistas/RevistaEstabilidadFinanciera/10/ Nov/Fic/ref0519.pdf y consultado: 28/01/2015.

Millán, J. R., Fruet, J. V. y Jimber, J. A. (2013). Análisis de Márgenes, Rentabilidad, Eficiencia y Productividad de la Banca Cooperativa Española. Disponible en: http:/helvia.uco.es/xmlui/bitstream/handle/10396/10889/jr6. pdf?sequence=1 y consultado: 08/05/2017.

Morón, E. (2003). Sistema de Alerta Temprana de Fragilidad Financiera. Disponible en: http://www.bcu.gub.uy/ autoriza/peiees/jor/2003/iees03j3270803.pdf y consultado: 02/02/2009.

Murillo, J. A. (2005). La Banca en México: Privatización, Crisis y Reordenamiento. Disponible en: http://www. cemla.org/old/pdf/red/RED_VII_MEXICO-Jose-Murillo.PDF y consultado: 16/03/2009. 
Redondo, D. y J. M. Rodríguez, (2014). Crisis en las entidades de crédito españolas: Un estudio mediante análisis discriminante. Estudios de Economía Aplicada, 32(2), 617-644. Disponible en: https://dialnet.unirioja.es/descarga/articulo/4680156.pdf y consultado: 25/02/2017.

Saavedra, M. L. (2008). La crisis financiera estadounidense y su impacto en la economía mexicana. Disponible en: http://iies.faces.ula.ve/revista/Articulos/Revista_26/Pdf/Rev26Saavedra.pdf y consultado: 31/10/2014.

Salvador, M. (2000). Análisis Discriminante, [en línea] 5campus.com, Estadística. Disponible en: http://www. 5campus.com/leccion/discri y consultado: 16/09/2007.

Turrent, E. (2003). Historia Sintética de la Banca en México, Banco de México. Disponible en: http://www.banxico.org.mx/sistema-financiero/material-educativo/basico/\%7BFFF17467-8ED6-2AB2-1B3B-ACCE5C2AF0E6\%7D.pdf y consultado: 12/01/2012.

\section{Tesis}

Carvallo, G. (2007). Medición de la Fortaleza Financiera de las Empresas: Clasificación según el Riesgo Crediticio y Estimación de la Probabilidad de Incumplimiento, Tesis de Maestría en Finanzas, Programa de Posgrado en Ciencias de la Administración, Universidad Nacional Autónoma de México. 